\title{
Effects of Navier slip on a steady flow of an incompressible viscous fluid confined within spirally enhanced channel
}

\author{
J. A. Gbadeyan 1', J. U. Abubakar ${ }^{1 *}$ and T. L. Oyekunle ${ }^{2}$
}

\author{
* Correspondence: abubakar.ju@ \\ unilorin.edu.ng \\ ${ }^{1}$ University of Ilorin, Ilorin, Kwara \\ State, Nigeria \\ Full list of author information is \\ available at the end of the article
}

\begin{abstract}
Investigation of the effect of slip on natural convective flow and heat transfer of a viscous incompressible fluid confined within a channel made up of a long vertical wavy wall and a parallel flat wall is carried out in this article. It is assumed that at the flat wall, there exists the slip condition. The coupled non-linear differential equations governing the fluid flow subjected to the relevant boundary conditions were perturbed and the resulting zero- and first-order set of equations were solved, using Adomian decomposition technique with the MAPLE 18 software. A comparison between the present study and an earlier one not involving a slip parameter and for which a different solution technique was used is carried out and the results are found consistent. The effects of various parameters involved in the problem viz Grashof number, slip parameter, heat source parameter, and wavelength parameter on the zero- and first-order temperature profile, velocity profile, skin friction, and Nusselt number at the walls are presented graphically and discussed quantitatively.
\end{abstract}

Keywords: Free convective, Heat transfer, Navier slip, Spirally enhanced channel Math Subject Classification: 76 W05, 76 M25, 76D05

\section{Introduction}

Nowadays, there exists an increasing number of flow devices of various geometrics through which fluids flow. This is perhaps, due to various new developments in science and technology. Traditionally, the boundary condition for the velocity field of a fluid at a solid surface of a medium through which the fluid flows is assumed equal to the velocity of the fluid at the solid surface. For instance, the value of such velocity is taken to be zero if the solid surface is assumed stationary. Meaning that the condition at a solid wall of the medium through which the fluid flows is referred to as the "no-slip" boundary condition. This no-slip condition has been experimented and proven satisfactorily only for macroscopic fluids where cohesion is weaker than adhesion. In other words, no-slip condition holds for fluid whose particles are found near the solid surfaces which fail to move with the flow [1]. However, recent experiments have shown that flow in micro-channels, for example, slip along repelling water (hydrophobic) walls obtained by coating the original non-hydrophobic walls. In particular, slip may

(c) The Author(s). 2020 Open Access This article is licensed under a Creative Commons Attribution 4.0 International License, which permits use, sharing, adaptation, distribution and reproduction in any medium or format, as long as you give appropriate credit to the original author(s) and the source, provide a link to the Creative Commons licence, and indicate if changes were made. The images or other third party material in this article are included in the article's Creative Commons licence, unless indicated otherwise in a credit line to the material. If material is not included in the article's Creative Commons licence and your intended use is not permitted by statutory regulation or exceeds the permitted use, you will need to obtain permission directly from the copyright holder. To view a copy of this licence, visit http://creativecommons.org/licenses/by/4.0/. 
have significant physical consequences for some microscale flows such as flows in the micro-electro-mechanical system (MEMS). Moreover, spillage at the micro-device walls through which fluid flows may produce an appreciable effect on the heat and mass transfer in the system. Several works which have been carried out on fluid slip phenomena for flows in various micro and non-micro devices include that in [2].

Interestingly, a theoretical discussion on slip phenomena had been earlier presented by Navier [3] who proposed a general (since it includes both no-slip and slip conditions) boundary condition. Specifically, Navier proposed that the velocity of the fluid at the solid wall, $U_{x}$, is linearly proportional to the shear stress at the wall i.e., $U_{x}=\beta\left(\frac{\partial V}{\partial y}\right)$ such that $\beta$ (the slip parameter) equals to zero implies the no-slip condition, while $\beta \neq 0$ (but finite) implies the occurrence of slip. Recently, Adesanya and Gbadeyan [4] used the Adomian decomposition method to examine the steady flow of visco-elastic fluid through a planar channel with slip condition. Halima et al. [5] investigated a fluid flow along a vertical porous channel with heat and mass transfer flow of a viscous and radiating fluid. They observed that the rise in the slip parameter slows down the fluid flow process. An unsteady MHD nonNewtonian fluid flows with the influence of radiation, heat transfer, and slip effect in a porous medium was investigated by Gbadeyan and Dada [6]. They found that a decrease in either Prandtl number or radiation parameter results in an increase in the temperature field while the velocity profile decreases as either radiation parameter $N$ or Grashof number $G$ decreases. Makinde [7] modelled an unsteady MHD flow and heat transfer with Navier slip and Newtonian heating toward a flat plate, and the result states that unsteadiness parameter affect the heating correspondingly while the thermal boundary layer thickness, local skin friction, and the heat transfer at the plate surface were seen to change noticeably due to the slip parameter. Mahmoud and Waheed [8] considered a micro-polar fluid over a heated stretching sheet coupled with heat generation/absorption and slip effect on MHD mixed convective flow. They highlighted that both velocity and temperature fields were appreciably affected by the effect of the mixed convective parameter. This effect of the slip was also investigated by Martin and Boyd [9] on the momentum and heat transfer in laminar boundary layer flow.

Moreover, while the problems of viscous fluid flowing over a corrugated wall finds its application in various areas such as cooling of re-entry vehicles, film vaporization in combustion chamber, rocket boosters, transpiration, and cross-heating on ablative surfaces, relatively few work has been carried out on the natural convective heat transfer in a viscous fluid flow located within a channel made up of two walls both of which are spirally enhanced. The problems of the flow of an incompressible viscous fluid through a long vertical channel made up of corrugated and parallel non-corrugated walls were studied by Vajrarelu and Sastri [10]. The study focused on heat transfer and flow characteristics. Fasogbon in [11] studied the effects of adiabatic wall waviness on the flow properties due to action of buoyancy between two long vertical walls, one of which is wavy and thermally insulated and the other being non-wavy but kept at a constant temperature. It was found that waviness of one of the walls produced a significant effect on both velocity and temperature profiles. Fasogbon and Omolehin [12] examined the radiation effect on natural convection in an irregular channel. They concluded that an increase in the magnitude of the fluid velocity of zeroth order and the Grashof number increases across the entire channel width. Meanwhile, an analytical solution of the two-dimensional corrugated channel was presented by Fasogbon [13] to study the heat and mass transfer by convection. It was argued that the heat source parameter has an 
accelerated effect on the fluid temperature. Natural convection temperature-dependent heat source in MHD and heat transfer with radiation in a porous medium between vertical wavy walls was investigated by Dada and Disu [14]. They pointed out that velocity rises with an increase in the value of porosity parameter, Grashof number, and radiation parameter, while it decreases with a rise in the heat source and magnetic parameter.

Recently, Gbadeyan et al. [15] analyzed the free convective heat and mass transfer flow of chemical reaction and thermal-radiation effects through an irregular vertical channel with constant volumetric heat generation/absorption. The result shows that a rise in the chemical reaction and radiation parameters decelerates the fluid velocity across the entire width of the channel. Soret and Dufour effects on heat and mass transfer in chemically reacting MHD flow through a rough channel were investigated by Gbadeyan et al. [16].

However, in all these previous studies, (except for Teneja and Jain [17], who claimed to have studied MHD flow with slip effects and temperature-dependent heat source in a viscous incompressible fluid confined between a long vertical wavy wall and a parallel flat wall. However, no specific result about the influence of slip is presented) the effect of slip on the wavy problem is neglected. Nevertheless, in many practical applications, the particles adjacent to a solid surface no longer take up the velocity of the surface. It, instead, has a finite tangential velocity, i.e., it slips along the surface $[18,19]$. Spectral relaxation method was used by Younghae et al. [20] to investigate the Navier's slip condition on time-dependent DarcyForchheimer nanofluid where unsteadiness parameter was seen to have a significant effect on velocity, temperature, concentration fields, the associated heat, and mass transport rates.

Therefore, the primary focus of this paper is to investigate the effect of slip at the parallel flat wall of free convective heat transfer in a viscous incompressible fluid confined within one wavy wall and one flat parallel wall. The non-dimensional boundary value problem governing the fluid flow was perturbed and the resulting zeroth- and first-order boundary value problems were solved, using Adomian decomposition method with the MAPLE 18 software.

\section{Model formulation}

A channel made up of one wavy wall and one parallel flat wall was considered. The $x$-axis is taken vertically upward, while the $y$-axis is taken perpendicular to it. The wavy wall and the flat wall are represented by $y=\epsilon^{*} \operatorname{coskx}$ and $y=d$ respectively as shown in Fig. 1 .
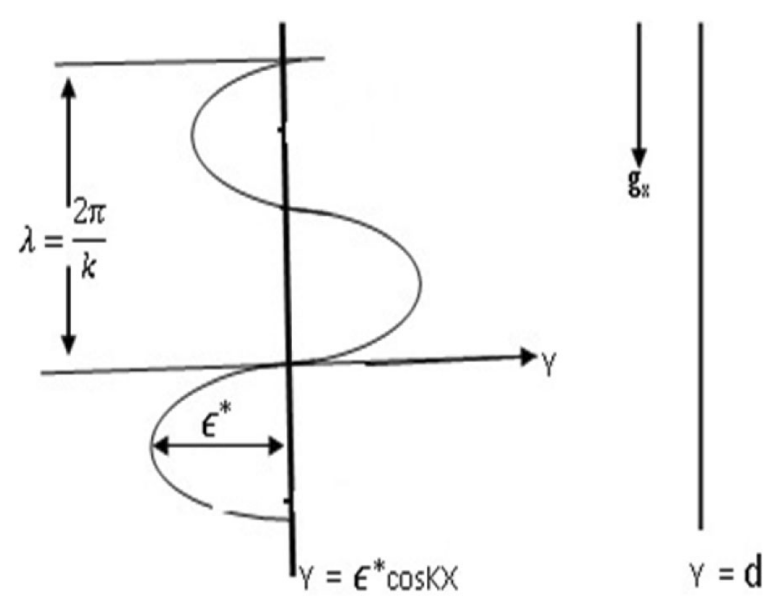

Fig. 1 The flow channel 
The flow is assumed to be laminar, steady, and two dimensional with slip at the parallel flat wall and that the wavy wall temperature is maintained at $T_{\mathrm{w}}$, while that of the parallel flat wall is at $T_{1}$. Also, the viscous dissipation and the work done by the pressure are assumed sufficiently small in comparison with both the heat flow by conduction and the wall temperatures. Furthermore, it is assumed that the wavelength of the wavy wall proportional to $\frac{1}{k}$ is large and that the volumetric heat source/sink term in the energy equation is constant. Based on these assumptions, the equations governing steady two-dimensional fluid flow and heat transfer made up of the momentum, continuity, and energy equations describing the flow are written as

Momentum equations

$$
\begin{aligned}
& \rho\left(U \frac{\partial U}{\partial X}+V \frac{\partial U}{\partial Y}\right)=-\frac{\partial P^{*}}{\partial X}+\mu\left(\frac{\partial^{2} U}{\partial X^{2}}+\frac{\partial^{2} U}{\partial Y^{2}}\right)-\rho g_{x} \beta\left(T-T_{s}\right) \\
& \rho\left(U \frac{\partial V}{\partial X}+V \frac{\partial V}{\partial Y}\right)=-\frac{\partial P^{*}}{\partial Y}+\mu\left(\frac{\partial^{2} V}{\partial X^{2}}+\frac{\partial^{2} V}{\partial Y^{2}}\right)
\end{aligned}
$$

Continuity equation

$$
\frac{\partial U}{\partial X}+\frac{\partial V}{\partial Y}=0
$$

Energy equation

$$
\rho C_{p}\left(U \frac{\partial T}{\partial X}+V \frac{\partial T}{\partial Y}\right)=K\left(\frac{\partial^{2} T}{\partial X^{2}}+\frac{\partial^{2} T}{\partial Y^{2}}\right)+Q
$$

where $U$ and $V$ are the velocity components, $P^{*}$ is the pressure, $\rho g_{x}$ is the buoyancy term in the $x$-direction, $\mathrm{Q}$ is the constant heat addition/absorption, and the other symbols have their usual meanings. The boundary conditions relevant to the problem are taken as

$$
\begin{aligned}
& U=0, V=0, T=T_{w}, Y=\epsilon^{*} \operatorname{coskx} \\
& U=\gamma \frac{\partial U}{\partial Y}, V=0, T=T_{1}, Y=d
\end{aligned}
$$

We define the non-dimensional variables as

$$
x=\frac{X}{d}, y=\frac{Y}{d}, u=\frac{U d}{v}, v=\frac{V d}{v}, \theta=\frac{\left(T_{1}-T_{s}\right)}{T_{w}-T_{s}}, \bar{P}=\frac{P^{*}}{\rho\left(\frac{v}{d}\right)^{2}}, \in=\frac{\epsilon^{*}}{d}
$$

where $T_{s}$ is the fluid temperature in static condition. Using Eq. (7), we nondimensionalized Eqs. (1) to (4) and the boundary conditions in Eq. (5) and Eq. (6) to obtain the following:

Momentum equation

$$
u \frac{\partial u}{\partial x}+v \frac{\partial u}{\partial y}=-\frac{\partial \bar{P}}{\partial x}+\frac{\partial^{2} u}{\partial x^{2}}+\frac{\partial^{2} u}{\partial y^{2}}-\rho g_{x} \frac{d^{3}}{\rho v^{2}}
$$




$$
u \frac{\partial v}{\partial x}+v \frac{\partial v}{\partial y}=-\frac{\partial \bar{P}}{\partial y}+\frac{\partial^{2} v}{\partial x^{2}}+\frac{\partial^{2} v}{\partial y^{2}}
$$

Continuity equation

$$
\frac{\partial u}{\partial x}+\frac{\partial v}{\partial y}=0
$$

Energy equation

$$
P\left[u \frac{\partial \theta}{\partial x}+v \frac{\partial \theta}{\partial y}\right]=\frac{\partial^{2} \theta}{\partial x^{2}}+\frac{\partial^{2} \theta}{\partial y^{2}}+\alpha
$$

where $P=\frac{\mu}{k} C_{p}, \rho v=\mu$ and $\alpha=\frac{Q . d^{2}}{K\left(T_{w}-T_{s}\right)}$

The non-dimensional boundary condition is

$$
\begin{aligned}
& u=0, v=0, \theta=1, y=\epsilon \cos \lambda x \\
& u=\beta \frac{\partial u}{\partial y}, v=0, \theta=r, y=1
\end{aligned}
$$

In the static fluid,

$$
-\frac{\partial \bar{P}_{s}}{\partial x}-\frac{\rho_{s} g_{x} d^{3}}{\rho \mu^{2}}=0
$$

Using Eq. (14) in Eq.(8) we have

$$
u \frac{\partial u}{\partial x}+v \frac{\partial u}{\partial y}=-\frac{\partial\left(\bar{P}-\bar{P}_{s}\right)}{\partial x}+\frac{\partial^{2} u}{\partial x^{2}}+\frac{\partial^{2} u}{\partial y^{2}}-\frac{\left(\rho-\rho_{s}\right)}{\rho} \frac{g_{x} d^{3}}{v^{2}}
$$

But the well-known Boussinesq approximation is

$$
\frac{\left(\rho-\rho_{s}\right)}{\rho}=-\beta\left(T_{w}-T_{s}\right) \theta, G=\frac{d^{3} g_{x} \beta\left(T_{w}-T_{s}\right)}{v^{2}}
$$

Substituting these terms into Eq. (15), the latter becomes

$$
u \frac{\partial u}{\partial x}+v \frac{\partial u}{\partial y}=-\frac{\partial\left(\bar{P}-\bar{P}_{s}\right)}{\partial x}+\frac{\partial^{2} u}{\partial x^{2}}+\frac{\partial^{2} u}{\partial y^{2}}+G \theta
$$

\section{The solution of the governing equation}

\section{Perturbation method}

The flow velocity, temperature, and pressure fields are perturbed as follows:

$$
\left(\begin{array}{l}
u(x, y)=u_{0}(y)+\epsilon u_{1}(x, y) \\
v(x, y)=\epsilon v_{1}(x, y) \\
\bar{P}(x, y)=P_{0}(y)+\epsilon P_{1}(x, y) \\
\theta(x, y)=\theta_{0}(y)+\epsilon \theta_{1}(x, y),
\end{array}\right.
$$

where the perturbations $u_{1}, v_{1}, P_{1}$, and $\theta_{1}$ are small compared to the mean or the zeroth-orderer quantities

Using Eq. (17) on Eqs. (9) to (11) and (16), we obtained the set of zeroth-orderer perturbation. 


$$
\left(\begin{array}{l}
\frac{d^{2} u_{0}}{d y}+G \theta_{0}=0 \\
\frac{d^{2} \theta_{0}}{d y^{2}}=-\alpha
\end{array}\right.
$$

and this set of first-order perturbation

$$
\left\{\begin{array}{l}
u_{0} \frac{\partial u_{1}}{\partial x}+v_{1} \frac{d u_{0}}{d y}=-\frac{\partial P_{1}}{\partial x}+\frac{\partial^{2} u_{1}}{\partial x^{2}}+\frac{\partial^{2} u_{1}}{\partial y^{2}}+G \theta_{1} \\
u_{0} \frac{\partial v_{1}}{\partial x}=-\frac{\partial P_{1}}{\partial y}+\frac{\partial^{2} v_{1}}{\partial x^{2}}+\frac{\partial^{2} v_{1}}{\partial y^{2}} \\
\frac{\partial u_{1}}{\partial x}+\frac{\partial v_{1}}{\partial y}=0 \\
P\left[u_{0} \frac{\partial \theta_{1}}{\partial x}+v_{1} \frac{d \theta_{0}}{d y}\right]=\frac{\partial^{2} \theta_{1}}{\partial x^{2}}+\frac{\partial^{2} \theta_{1}}{\partial y^{2}}
\end{array}\right.
$$

with boundary conditions

$$
\begin{aligned}
& \left(\begin{array}{l}
u_{0}=0, \theta_{0}=1, y=0 \\
u_{0}=\beta \frac{d u_{0}}{d y}, \theta_{0}=r, y=1
\end{array}\right. \\
& \left(\begin{array}{l}
u_{1}=u_{0}^{\prime}, v_{1}=0, \theta_{1}=-\theta_{0}^{\prime}, y=0 \\
u_{1}=\beta \frac{\partial u_{1}}{\partial y}, v_{1}=0, \theta_{1}=0, y=1
\end{array}\right.
\end{aligned}
$$

\section{Introduction of stream function into the first-order problem}

For further simplification, the stream function $\bar{\psi}(x, y)$ defined by

$$
u_{1}=-\frac{\partial \bar{\psi}_{1}}{\partial y}, v_{1}=\frac{\partial \bar{\psi}_{1}}{\partial x}
$$

is then introduced into the set of first-order perturbation Eq. (19) to obtain

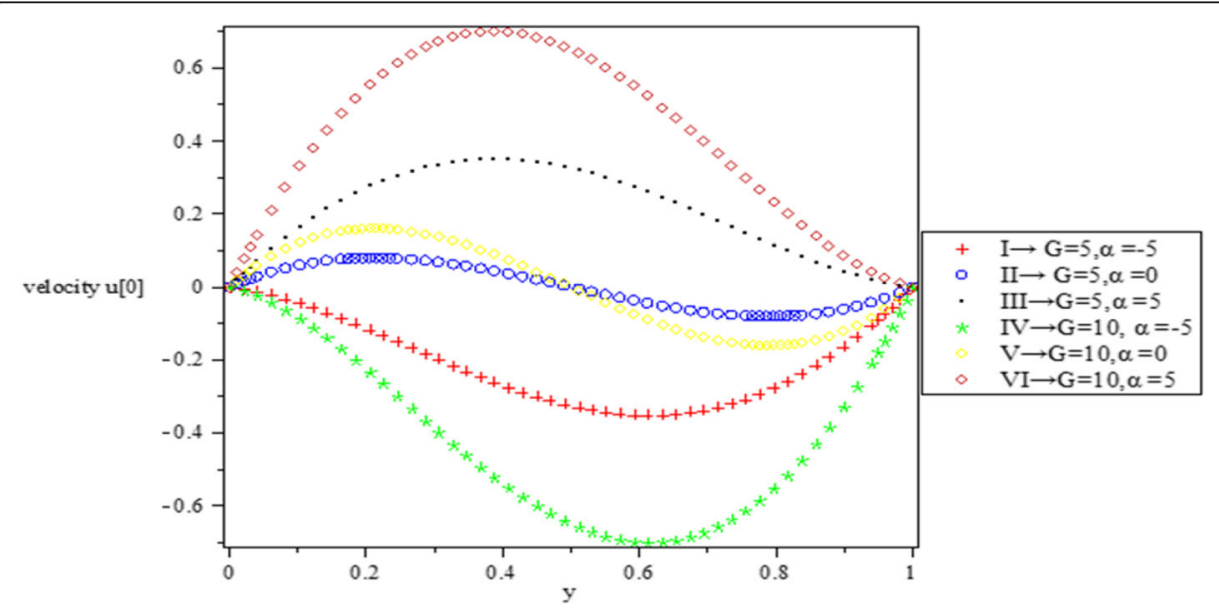

Fig. 2 Zeroth-order velocity (u[0] profile, $r=-1$ and $\beta=0$ ) 


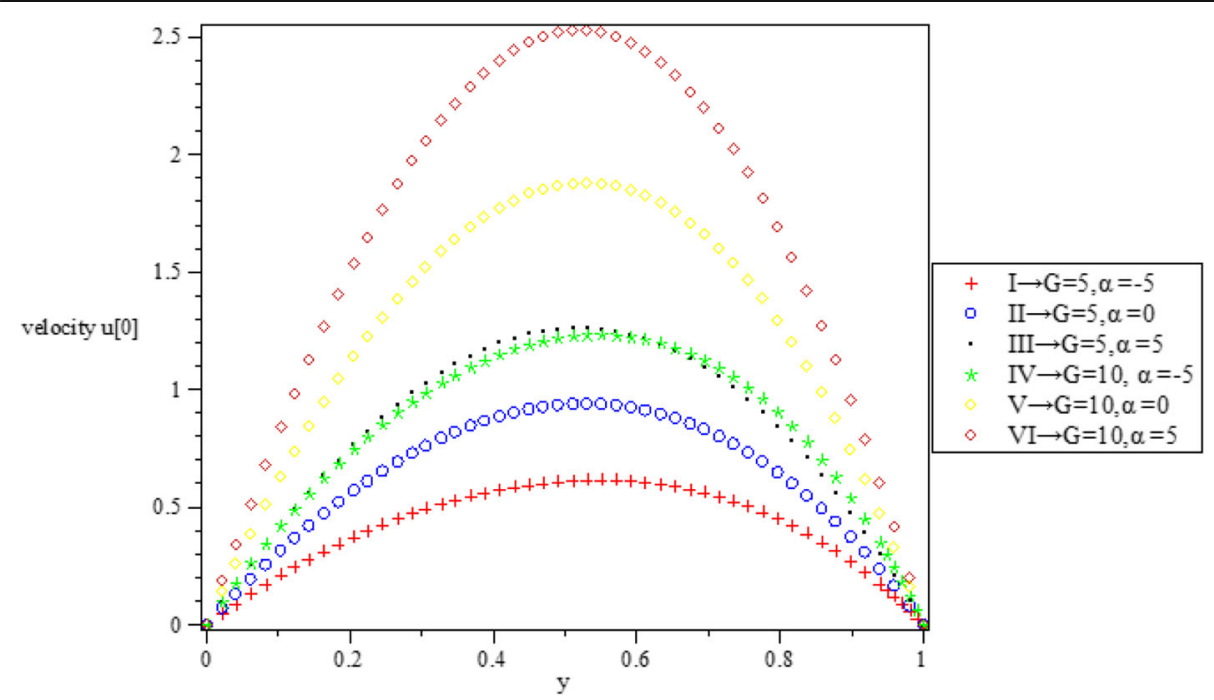

Fig. 3 Zeroth-order velocity (u[0] profile, $r=2$ and $\beta=0$ )

$$
\begin{aligned}
& u_{0}\left(\bar{\psi}_{1, x x x}+\bar{\psi}_{1, x y y}\right)-u^{\prime \prime}{ }_{0} \bar{\psi}_{1, x}=\bar{\psi}_{1, x x x x}+\bar{\psi}_{1, y y y y}+2 \bar{\psi}_{1, x x y y}-G \theta_{1, y} \\
& P\left[u_{0} \theta_{1, x}+\theta_{0}^{\prime} \bar{\psi}_{1, x}\right]=\theta_{1, x x}+\theta_{1, y y}
\end{aligned}
$$

Eqs. (22) and (23) are further simplified by the introduction of another form of solution which is wave-like due to the motion of fluid at the walls. We then assumed the solution to be

$$
\bar{\psi}_{1}(x, y)=\epsilon \exp ^{i \lambda x} \psi(y), \theta_{1}(x, y)=\epsilon \exp ^{i \lambda x} t(y)
$$

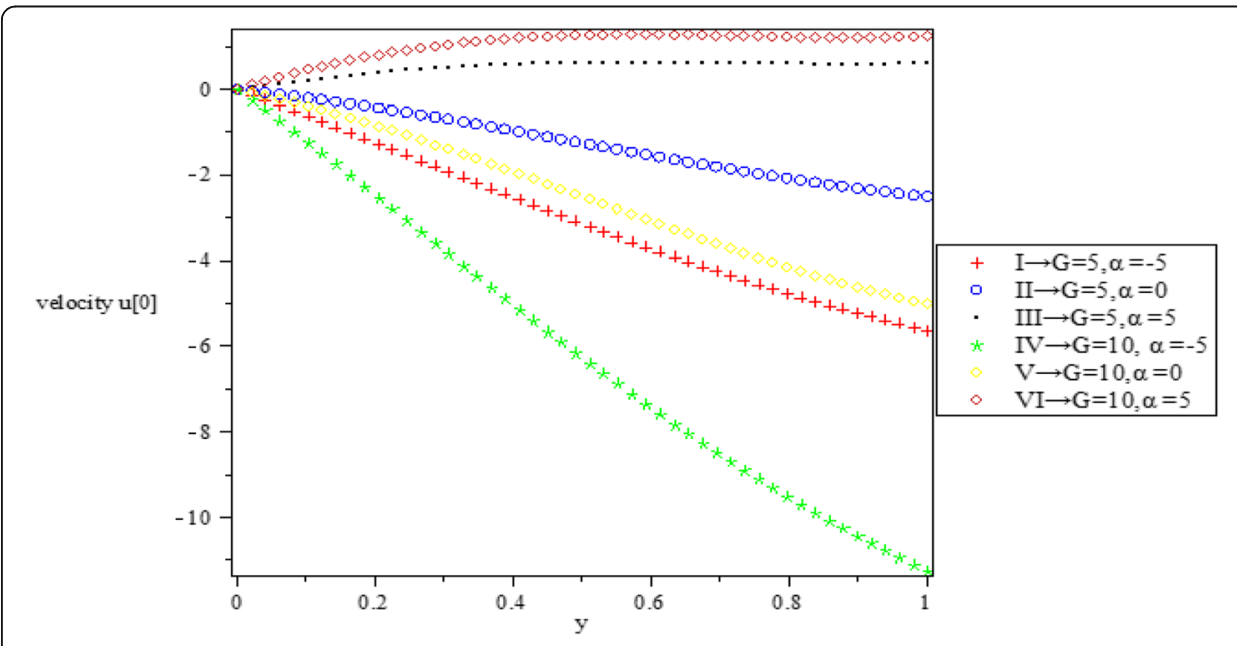

Fig. 4 Zeroth-order velocity ( $u[0]$ profile, $r=2$ and $\beta=1.5$ ) 


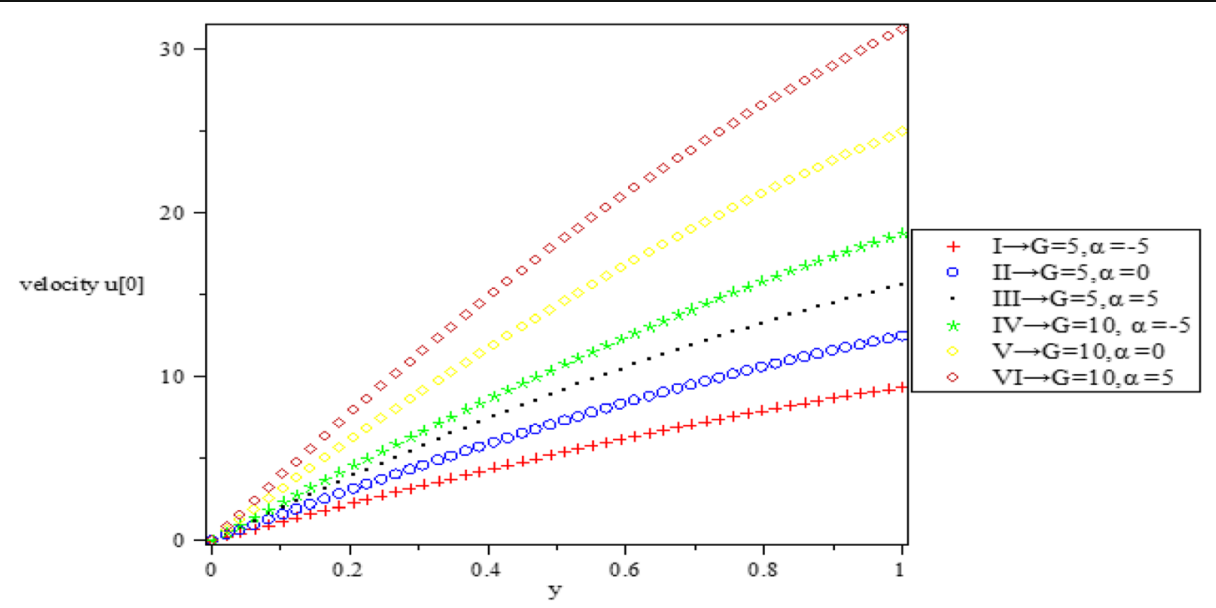

Fig. 5 Zeroth-order velocity (u[0] profile, $r=2$ and $\beta=1.5$ )

and

$$
\psi(\lambda, y)=\sum_{i=0}^{\alpha} \lambda^{i} \psi_{i}, t(\lambda, y)=\sum_{i=0}^{\alpha} \lambda^{i} t_{i}, i=0,1,2
$$

Using Eqs. (24) and (25) in Eqs. (22) and (23), we obtained the following set of ordinary differential equations and corresponding boundary conditions for various orders of $\lambda$.

Order of $\lambda^{0}$ we have

$$
\left(\begin{array}{l}
\psi_{0}^{i v}(y)=G t^{\prime}(y) \\
t^{\prime \prime}{ }_{0}(y)=0
\end{array}\right.
$$

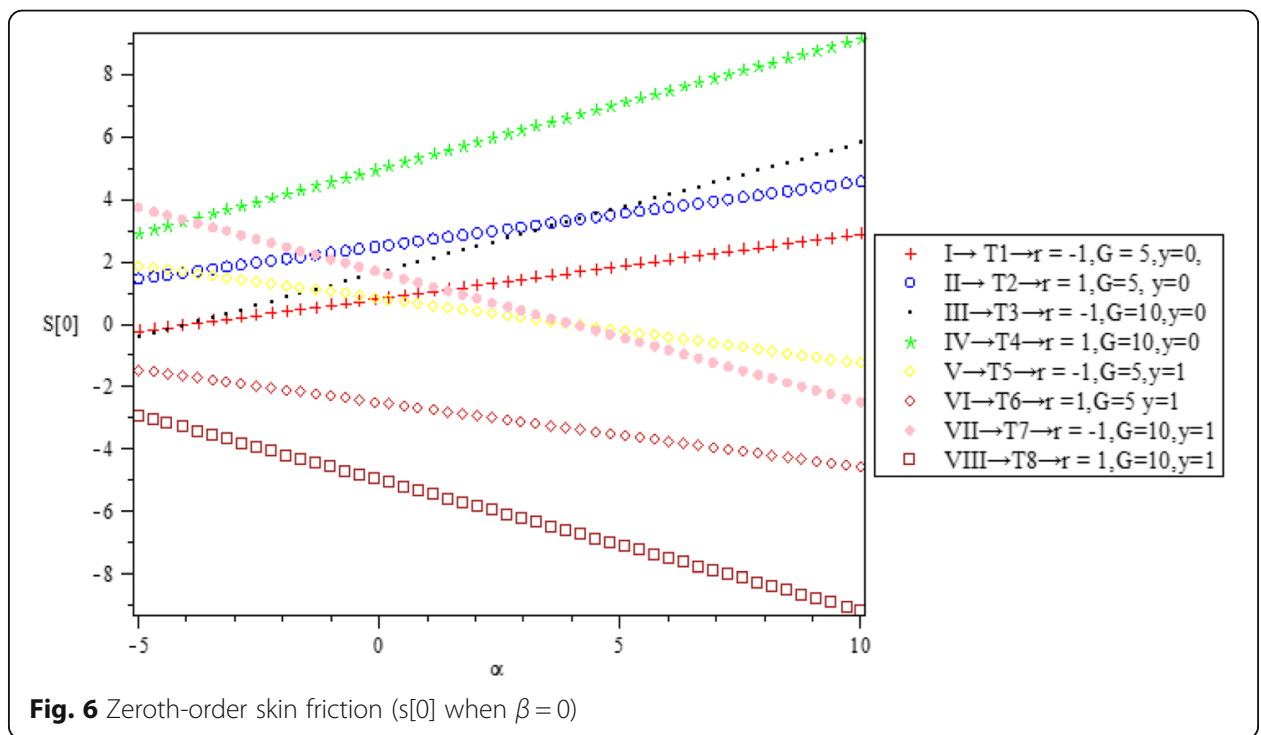

Fig. 6 Zeroth-order skin friction (s[0] when $\beta=0$ ) 


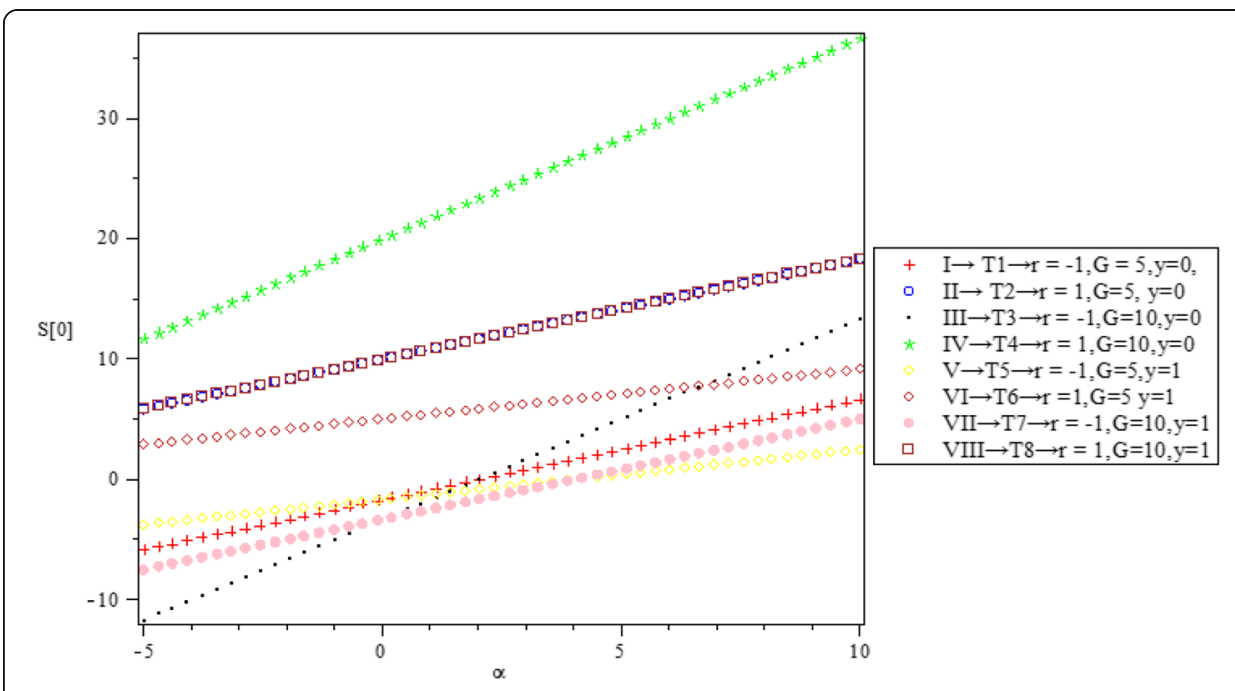

Fig. 7 Zeroth-order skin friction (s[0] when $\beta=1.5$ )

Order of $\lambda^{1}$

$$
\left(\begin{array}{l}
\psi_{1}^{i v}(y)+i u^{\prime \prime}{ }_{0}(y) \psi_{0}(y)-i u_{0}(y) \psi^{\prime \prime}{ }_{0}(y)=G t^{\prime}{ }_{1}(y) \\
t^{\prime \prime}{ }_{1}(y)=P i\left[u_{0}(y) t_{0}(y)+\theta^{\prime}{ }_{0}(y) \psi_{0}(y)\right]
\end{array}\right.
$$

Order of $\lambda^{2}$

$$
\left(\begin{array}{l}
\psi_{2}^{i v}(y)+i u^{\prime \prime}{ }_{0}(y) \psi_{1}(y)-i u_{0}(y) \psi^{\prime \prime}{ }_{1}(y)-2 \psi^{\prime \prime}{ }_{0}(y)=G t^{\prime}{ }_{2}(y) \\
t^{\prime \prime}{ }_{2}(y)=P i\left[u_{0}(y) t_{1}(y)+\theta^{\prime}{ }_{0}(y) \psi_{1}(y)\right]+t_{0}(y)
\end{array}\right.
$$

and boundary conditions

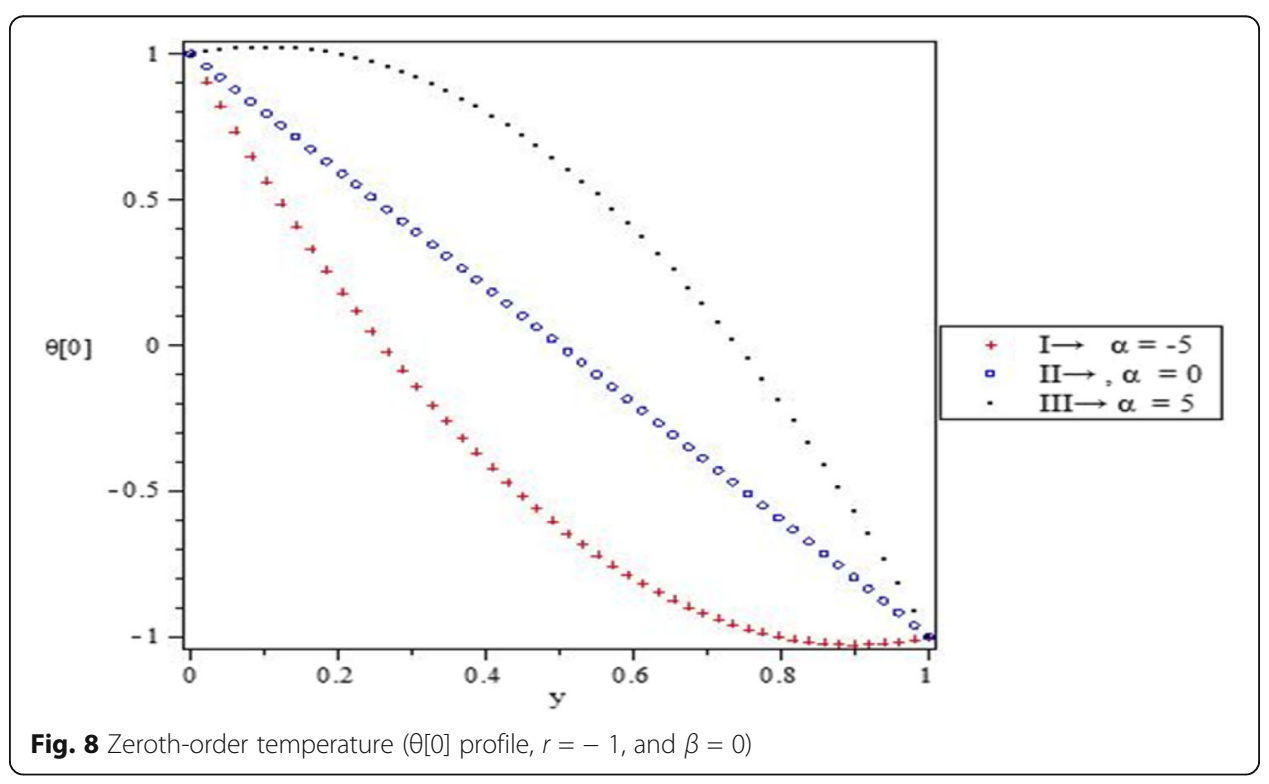




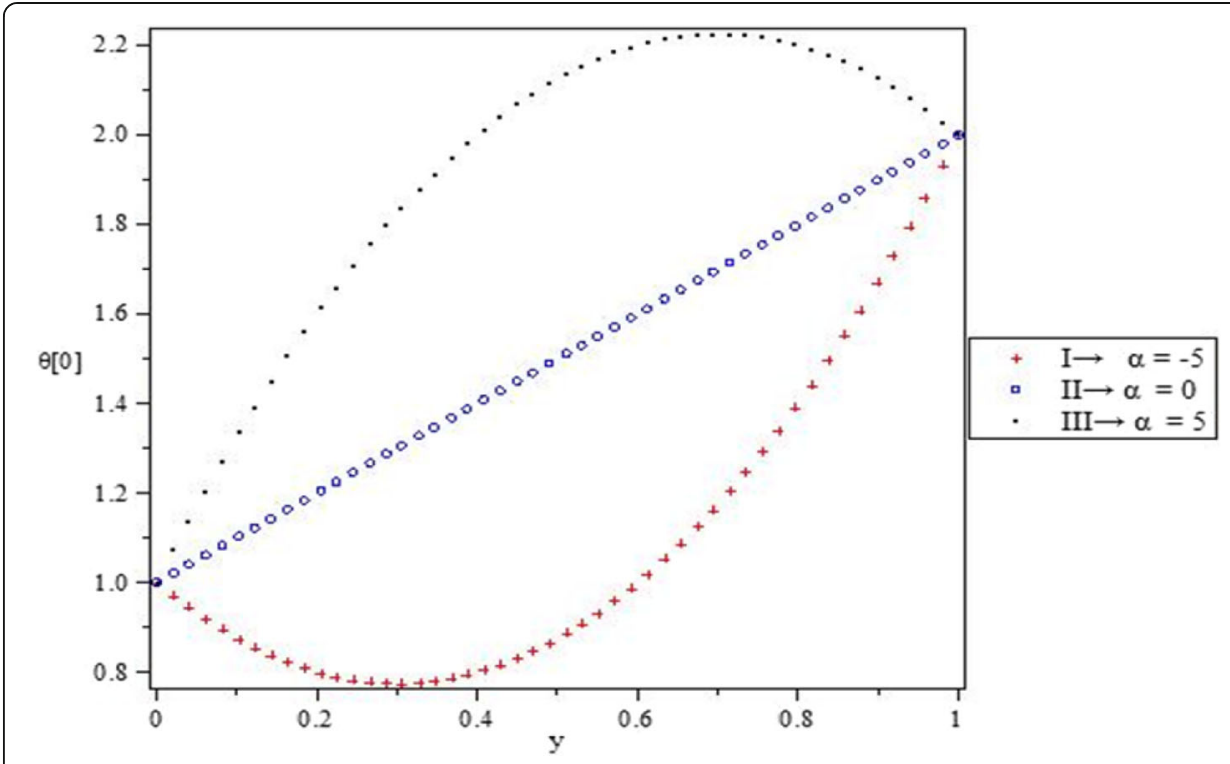

Fig. 9 Zeroth-order temperature ( $\theta[0]$ profile, $r=2$, and $\beta=0)$

$$
\begin{aligned}
& \left(\begin{array}{l}
\psi_{0}^{\prime}(y)=u_{0}^{\prime}, \psi_{0}(y)=0, t_{0}(y)=-\theta_{0}^{\prime} \text { on } y=0 \\
\psi_{0}^{\prime}(y)=\beta \psi^{\prime \prime}{ }_{0}, \psi_{0}(y)=0, t_{0}(y)=0 \text { on } y=0
\end{array}\right. \\
& \left(\begin{array}{l}
\psi_{i}^{\prime}(y)=0, \psi_{i}(y)=0, t_{i}(y)=0 \text { on } y=0 \\
\psi_{i}^{\prime}(y)=\beta \psi^{\prime \prime}{ }_{i}, \psi_{i}(y)=0, t_{i}(y)=0 \text { on } y=1, \forall i \geq 1
\end{array}\right.
\end{aligned}
$$

The Adomian decomposition method is then used to solve the zeroth-order (Eq. (18)) with boundary condition introduced in Eq. (20) and the first-order Eqs. (26), (27), and (28) with boundary conditions presented in Eqs. (29) and (30) using the Chen and $\mathrm{Lu}$ [21] program with Maple 18 to obtain the following expressions of $u_{0}$ and $\theta_{0}$ respectively,

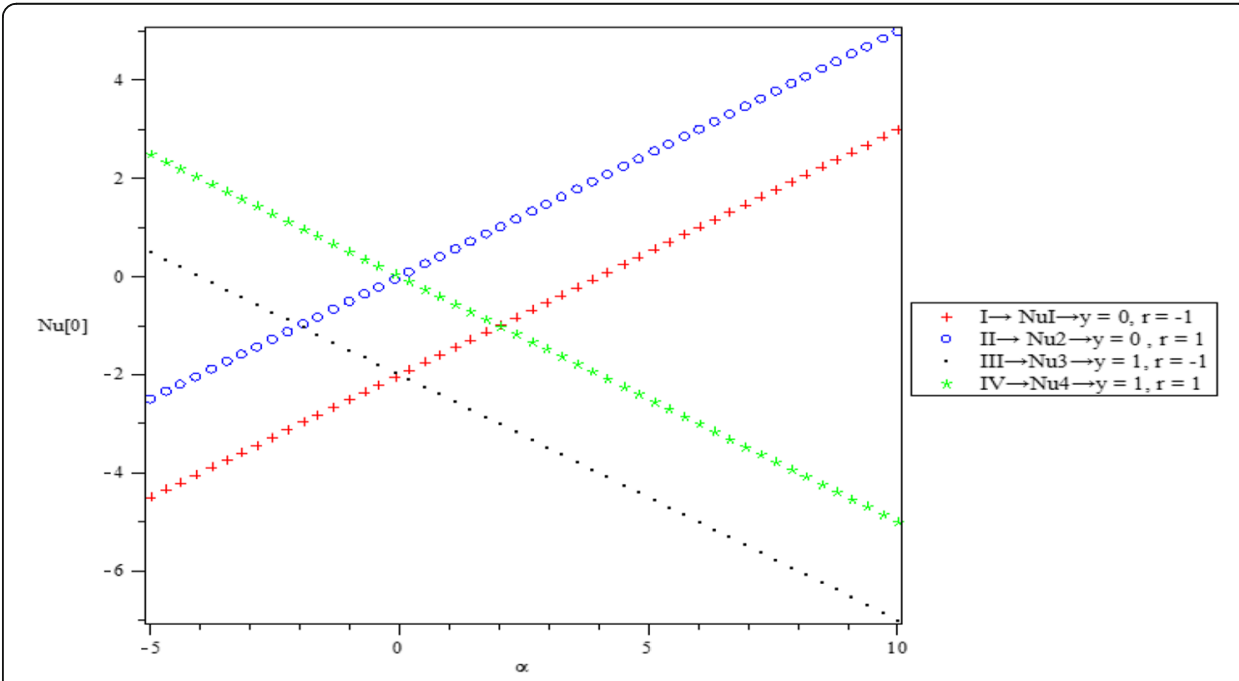

Fig. 10 Zeroth-order Nusset number ( $\mathrm{Nu}[0]$ when $\beta=0)$ 


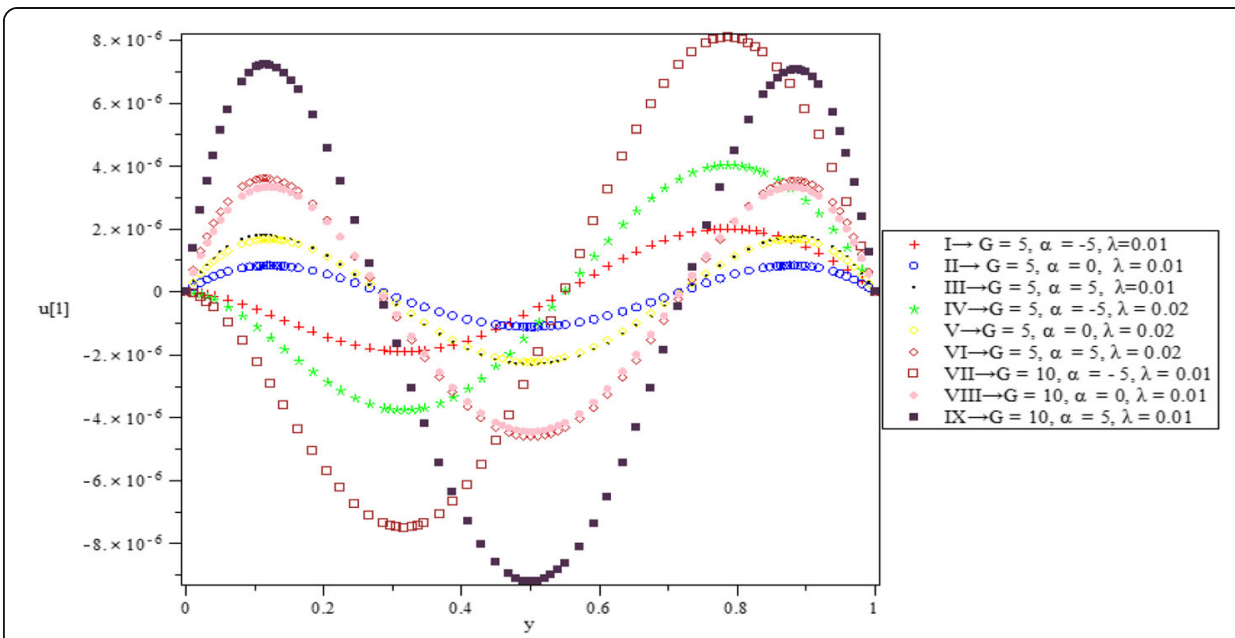

Fig. 11 First-order velocity (u[1] profile, $r=-1, \boldsymbol{\epsilon}=0.25, P=0.71$, and $\beta=0$ )

$$
\begin{aligned}
u_{0}= & \frac{1}{24} \frac{G(2 \beta \alpha+12 \beta r+12 \beta-8-\alpha-4 r) y}{\beta-1} \\
& +\frac{1}{24} G \alpha y^{4}-\frac{1}{6} G\left(r-1+\frac{1}{2} \alpha\right) y^{3}-\frac{1}{2} G y^{2} \\
\theta_{0}= & 1+\left(r-1+\frac{1}{2} \alpha\right) y-\frac{1}{2} \alpha y^{2}
\end{aligned}
$$

The expressions obtained for $u_{1}, v_{1}$, and $\theta_{1}$ are lengthy and are not presented here for brevity sake.

The zeroth-order skin friction and Nusselt number are expressed as

$$
\tau^{0}=u_{0}^{\prime}(y) \quad y=0
$$

and

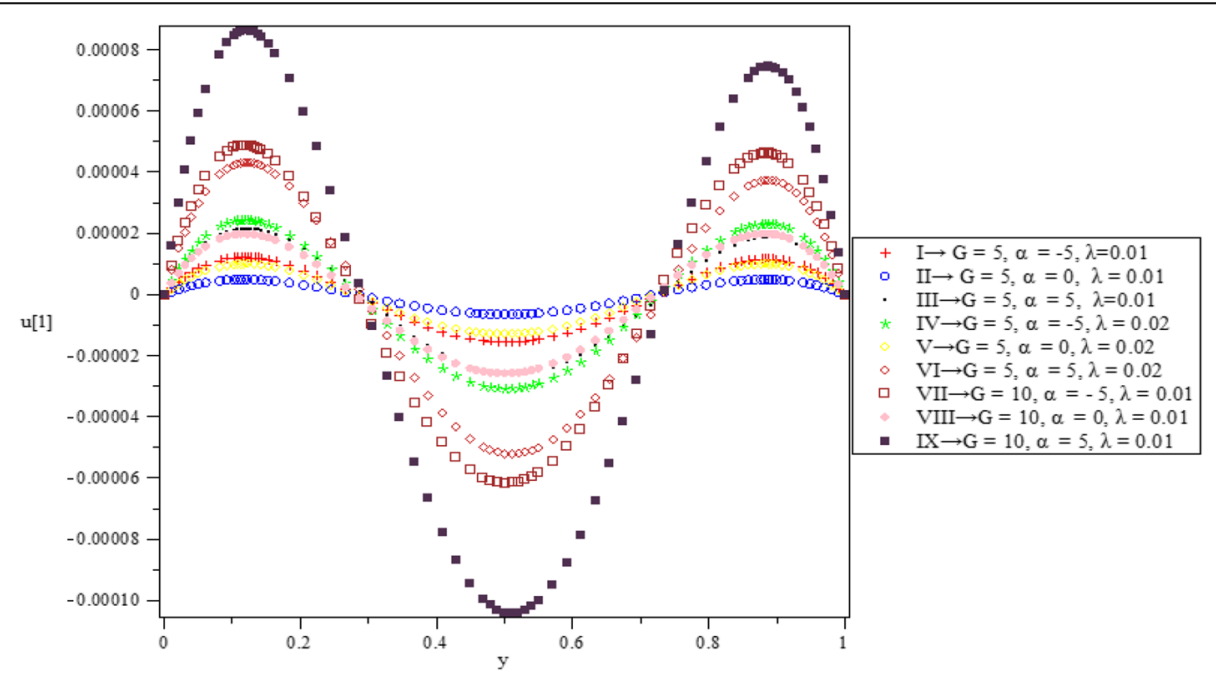

Fig. 12 First-order velocity (u[1] profile, $r=-1, \boldsymbol{\epsilon}=0.25, P=7$, and $\beta=0$ ) 


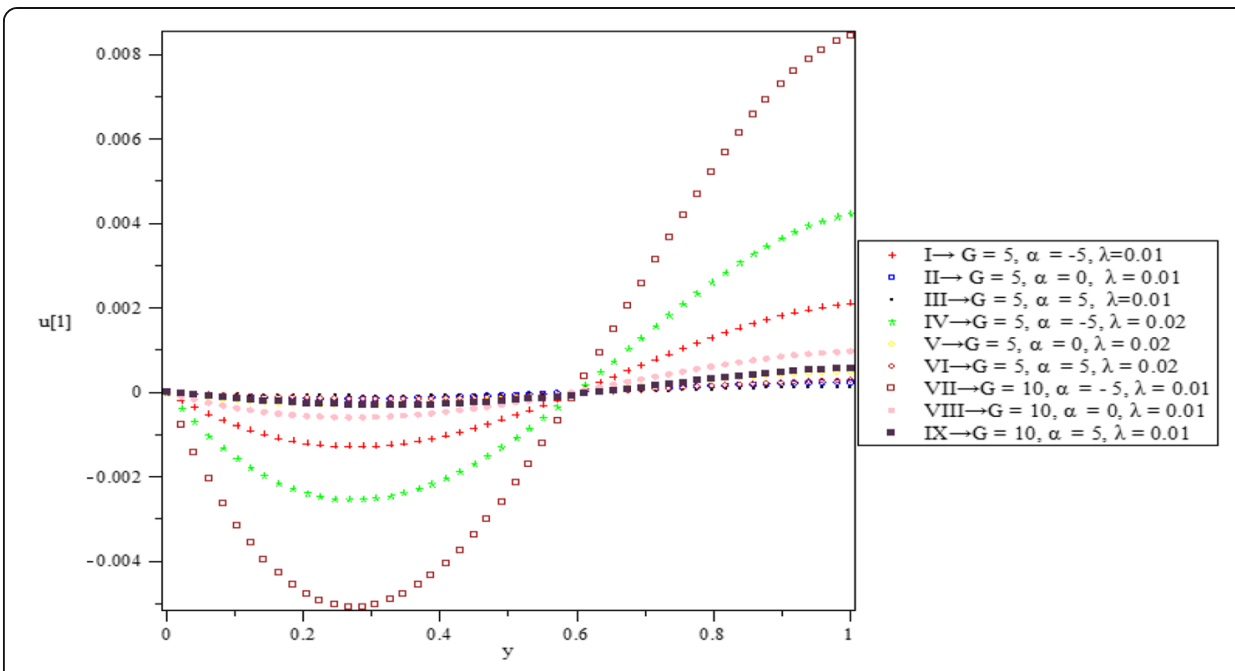

Fig. 13 First-order velocity (u[1] profile, $r=-1, \epsilon=0.25, P=0.71$, and $\beta=1.5$ )

$$
N u^{0}=\theta_{0}^{\prime}(y) \quad y=0
$$

While the first-order skin friction and Nusselt number are expressed as

$$
\tau^{1}=\epsilon\left[\psi^{\prime \prime}{ }_{i}(y) \sin \lambda x-\psi^{\prime \prime}{ }_{r}(y) \cos \lambda x\right]-\lambda^{2} \epsilon\left[\psi_{r} \cos \lambda x-\psi_{i}(y) \sin \lambda x\right]
$$

and

$$
N u^{1}=\epsilon\left[t_{r}^{\prime}(y) \cos \lambda x-t^{\prime}{ }_{i}(y) \sin \lambda x\right]
$$

\section{Discussions of results}

The main interest of the present work is to examine the influence of Navier slip $\beta$, in the presence of the parameters Grashof number $G$, heat source $\alpha$, and frequency $\lambda$, on the steady natural convective flow and heat transfer of viscous incompressible fluid confined within a spirally enhance channel. Hence, the velocity and

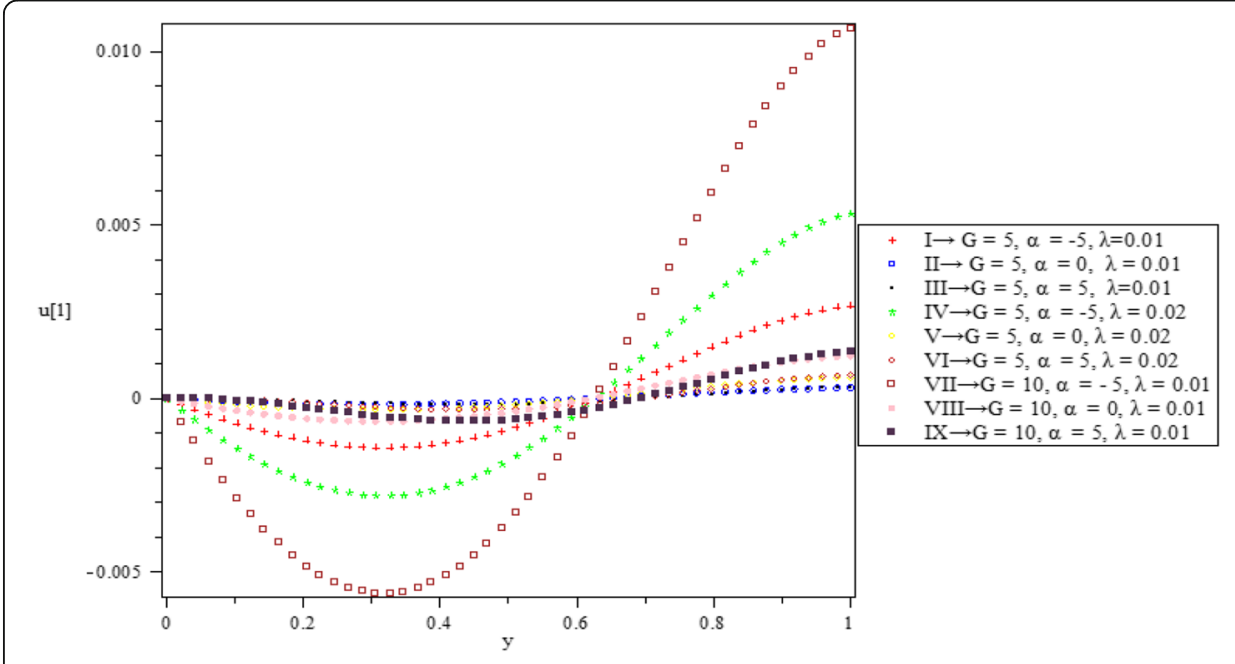

Fig. 14 First-order velocity (u[1] profile, $r=-1, \boldsymbol{\epsilon}=0.25, P=7$, and $\beta=1.5$ ) 


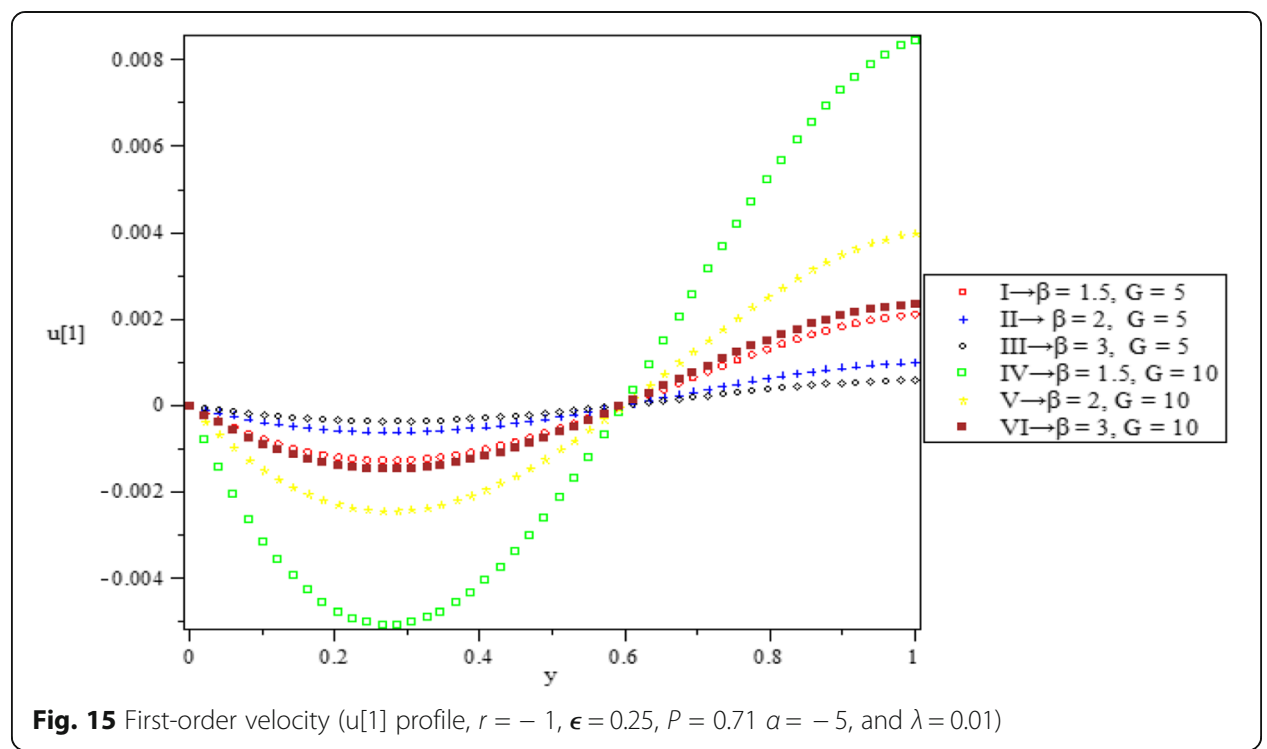

temperature profiles, as well as the skin friction and Nusselt number, were obtained by assigning physically realistic values to various parameters involved in the problem. For example, the Prandtl number $(P)$ is taken to be either 0.71 (air) or 7 (water), $\alpha=0$ signifies the absence of heat source, $\alpha<0$ denotes heat sink, while $\alpha>0$ represents the heat source. The positive value of $G$ corresponds to either cooling of the wall or heating of the fluid by natural convection currents. From a practical point of view, the wall temperature ratio $(r)$ takes on the value -1 and 2 . Physically, $r=-1$ means that the average temperature of the two walls equals to that of the static fluid, while $r=2$ means the wall temperatures are unequal. Geometrically, the spirality of the channel is denoted by the parameter $\epsilon>0$ which is however assumed small in this analysis. Specifically, $\epsilon=0.23$, while $\lambda=0.001$, 0.002 . All these values are obtained following $[13,16]$.

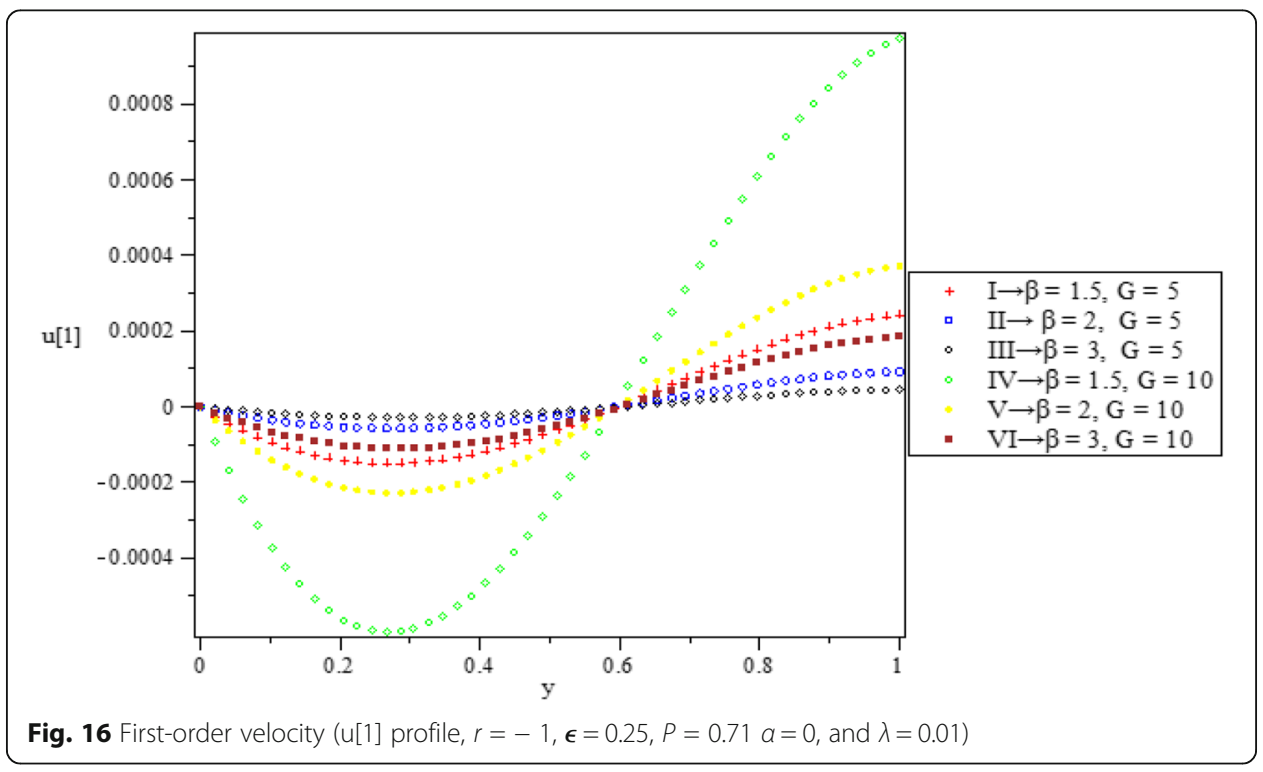




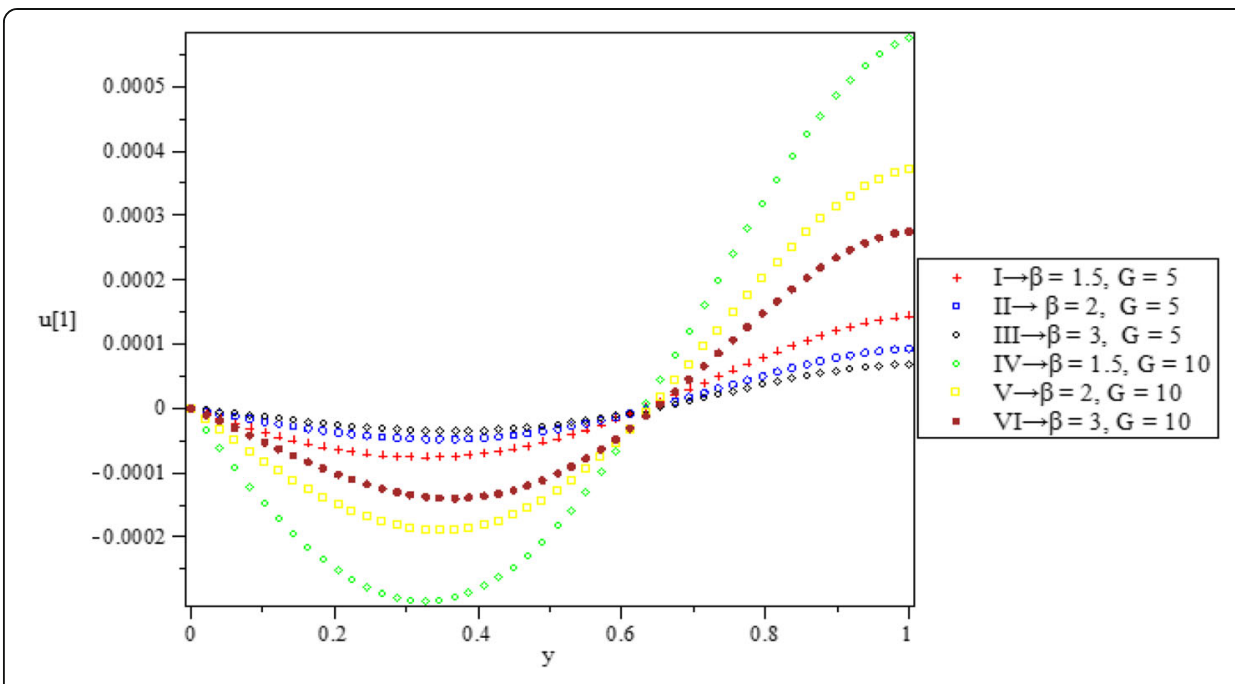

Fig. 17 First-order velocity (u[1] profile, $r=-1, \epsilon=0.25, P=0.71 a=5$, and $\lambda=0.01$ )

\section{Discussion of zeroth-order results}

Velocity profile

In this subsection, the influence of the various parameters $(G, \alpha, \lambda$, and $\beta)$ on the zeroth-order velocity $u_{0}$ of the fluid is discussed. This is carried out by starting with the comparison of the present result with the previous ones. To accomplish this, the new parameter $(\operatorname{slip} \beta)$ is set to zero.

The results in Figs. 2 and 3 show the velocity distribution profiles for $\beta=0$ (i.e., noslip condition) and when the wall temperature ratio $(r)$ is -1 and 2 respectively (i.e., $r=-1$ ). From Fig. 2, it is observed that increasing the Grashof number $(G)$ increases the fluid velocity when there is heat source (i.e., $\alpha>0$, see curves III and VI). This maybe due to the fact that an increase in thermal boyancy means a temperature

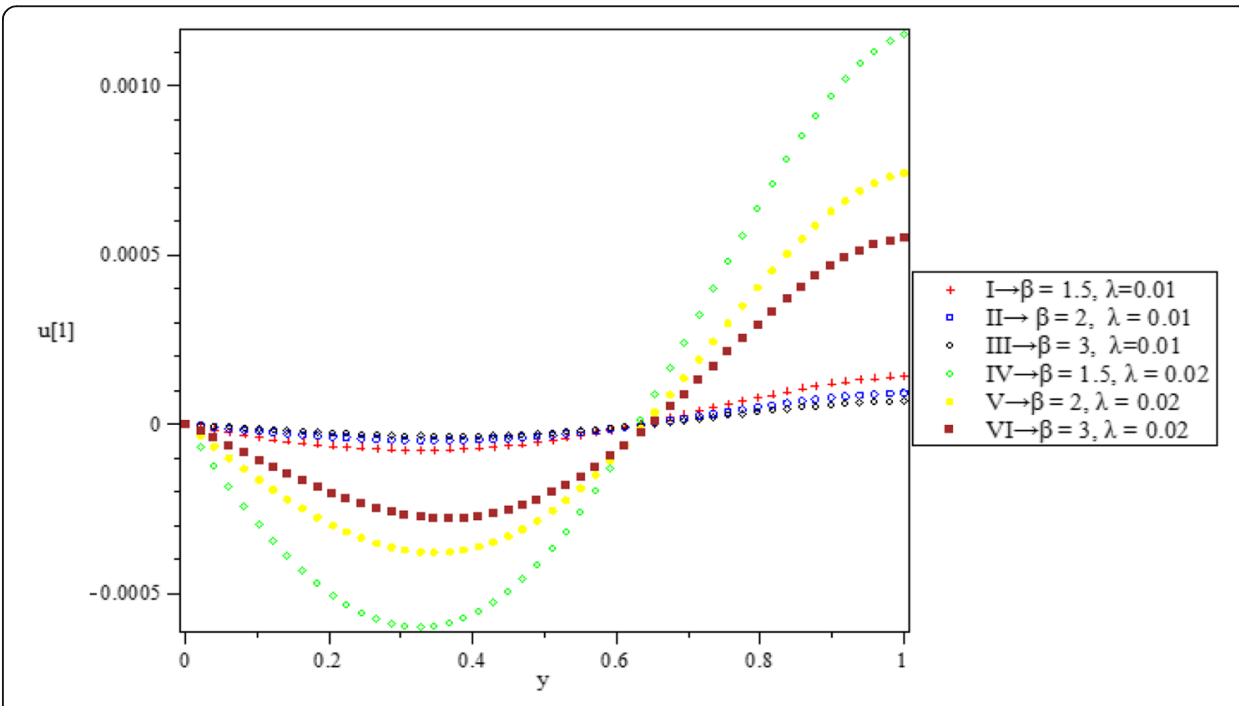

Fig. 18 First-order velocity (u[1] profile, $r=-1, \boldsymbol{\epsilon}=0.25, P=0.71 \quad a=5$, and $G=5$ ) 


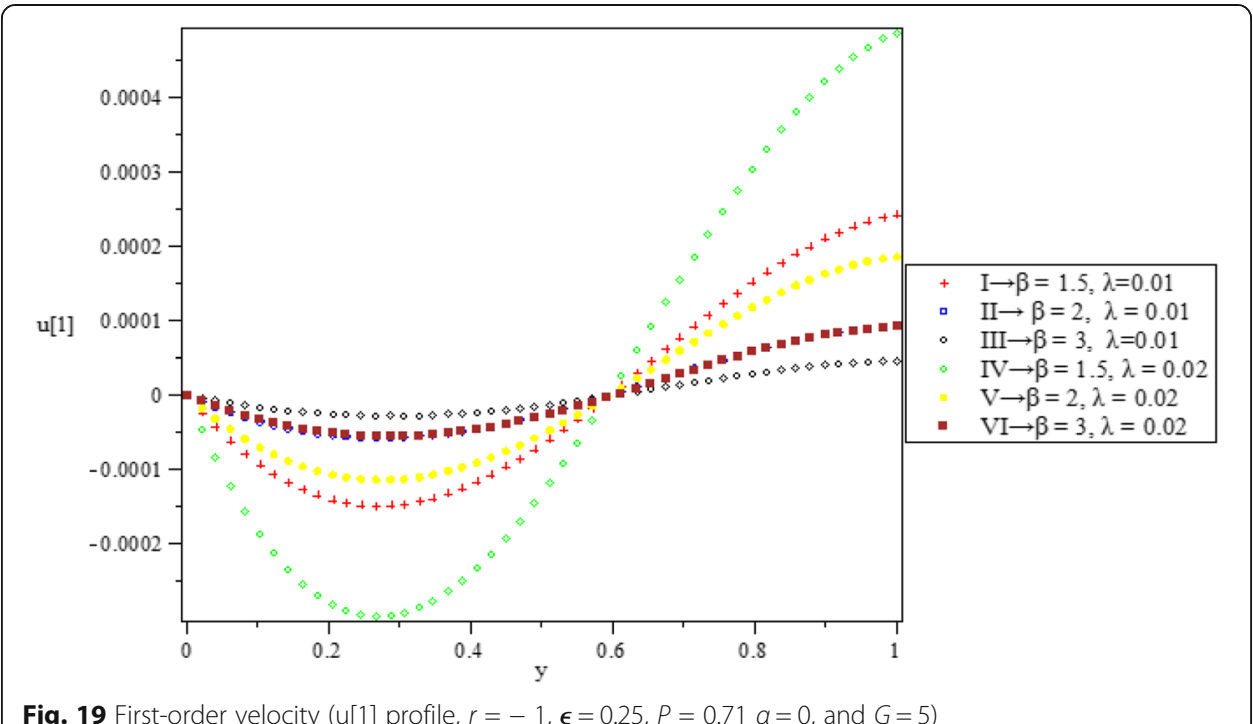

Fig. 19 First-order velocity ( $\mathrm{u}[1]$ profile, $r=-1, \epsilon=0.25, P=0.71 a=0$, and $G=5$ )

difference in the flow, which enhances fluid velocity. Also when there is heat sink (i.e., $\alpha<0$ ), the velocity decreases with an increase in Grashof number $(G)$ (see curves I and IV).

In the absence of heat source (i.e., $\alpha=0$ curves II and V) increase in Grashof number $(G)$, it increases the velocity of the fluid to half part of the channel then decreases. However, on fixing $G$ and varying the heat source parameter $(\alpha)$ from $\alpha=-5$ to $\alpha=0$ and then to $\alpha=5$, it is seen that there is a significant increase on the fluid velocity (see curves I, II, and III) when $r=-1$.

Figure 3 depicts the velocity distribution profile when $r=2$ and $\beta=0$ (i.e., no-slip condition); the fluid velocity $u_{0}$ increases generally as Grashof number increases for all values of $\alpha$. It is also observed that when $G$ is fixed and the heat source parameter $\alpha$ is increased from -5 to 5 , the velocity $u_{0}$ of the fluid increases generally across the channel width. For completeness sake, it is being remarked here that these results are in agreement with those in Vajravelu and Sastri [10].

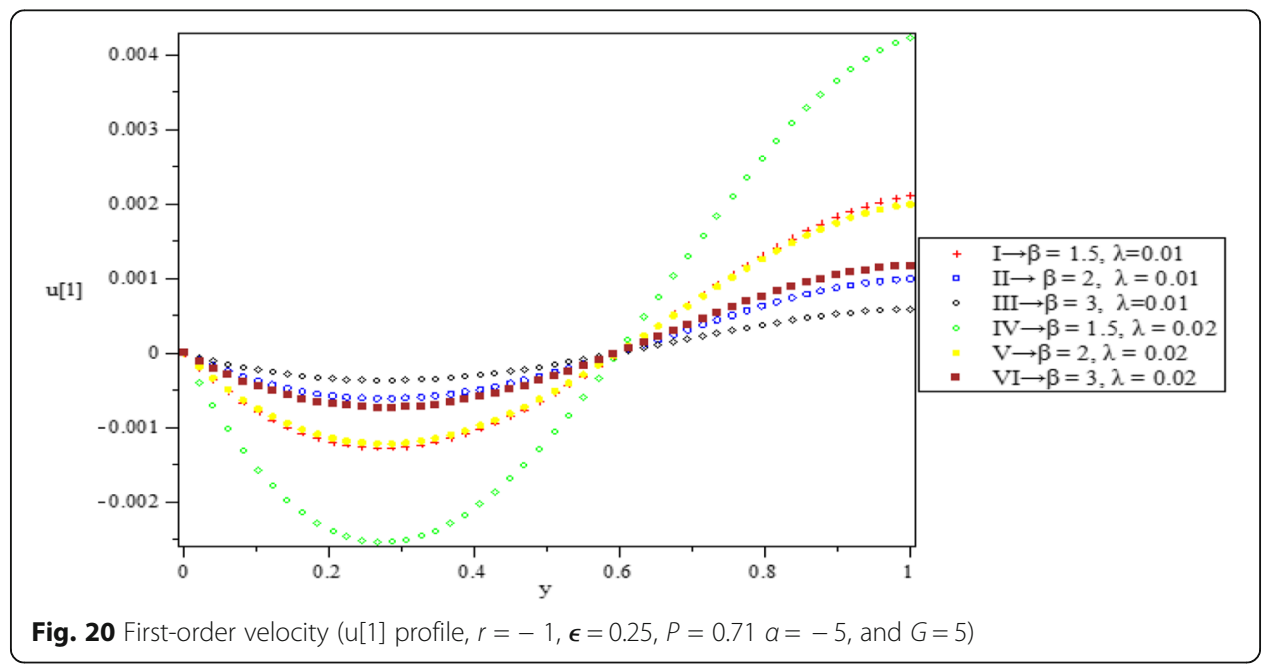




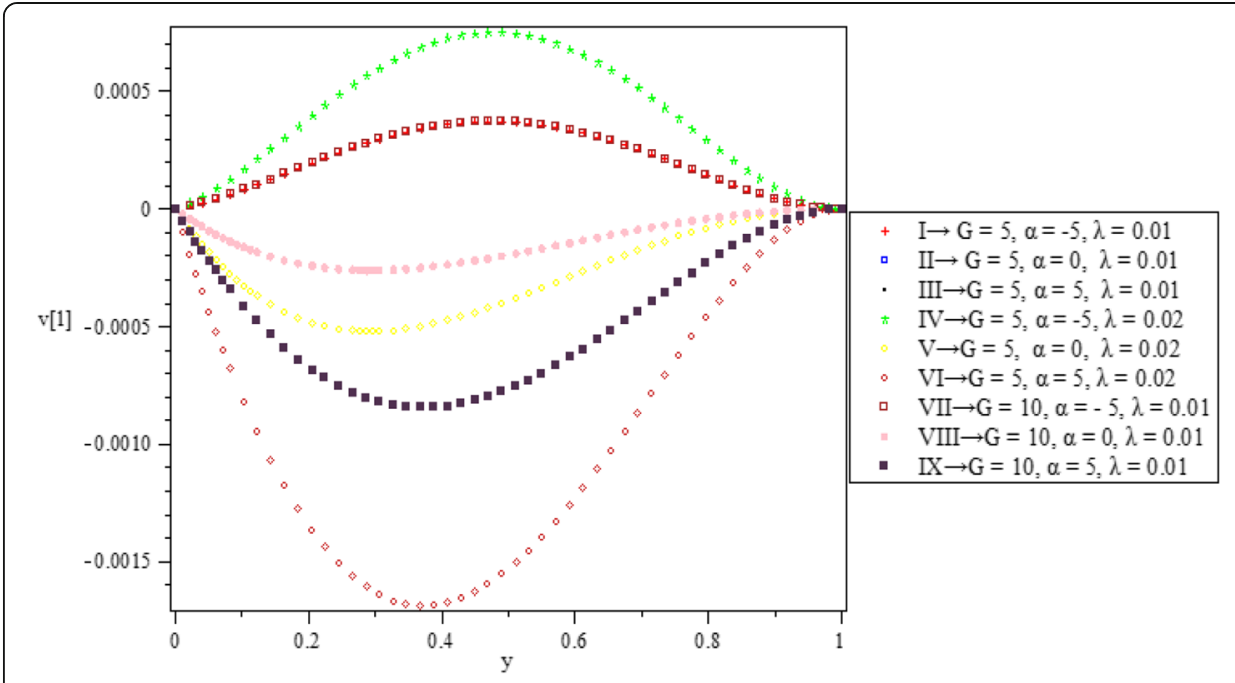

Fig. 21 First-order velocity (v[1] profile, $r=-1, \boldsymbol{\epsilon}=0.25, P=0.71$, and $\beta=0$ )

Figures 4 and 5 illustrate the effect of slip $(\beta)$ on the velocity distribution. It is observed from Fig. 4 that when $\beta=1.5$ and $r=-1$, the fluid velocity linearly decreases when Grashof number $(G)$ increases for $\alpha \leq 0$ (see curves I and IV, II and V) and it increases for $\alpha>0$ (see curves III and VI). Also, when $G$ is fixed and the heat source parameter $(\alpha)$ is varied from $-5,0$, and 5 , the fluid velocity increases linearly (see curves I, II, and III). While it is seen from Fig. 5 that when $\beta=1.5$ and $r=2$, the fluid velocity linearly increases as the Grashof number $(G)$ increases in all cases.

The zeroth-order skin friction at slip $(\beta=0)$ is shown in Fig. 6 and it is seen that at either wall is a linear function of the heat source parameter $\alpha$. The skin friction at the wavy wall $y=0$ increases as the heat source parameter $\alpha$ increases, and at the flat wall $y=1$, it decreases as the $\alpha$ increases. It is also, observed that the skin friction increases generally at $y=0$ as $G$ increases, while at $y=1$, it decreases as $G$ increases, for any value of wall temperature ratio $r$. While Fig. 7 depicts zeroth-order skin friction when slip $(\beta)=1.5$, it shows that the skin friction increases linearly as the heat source parameter

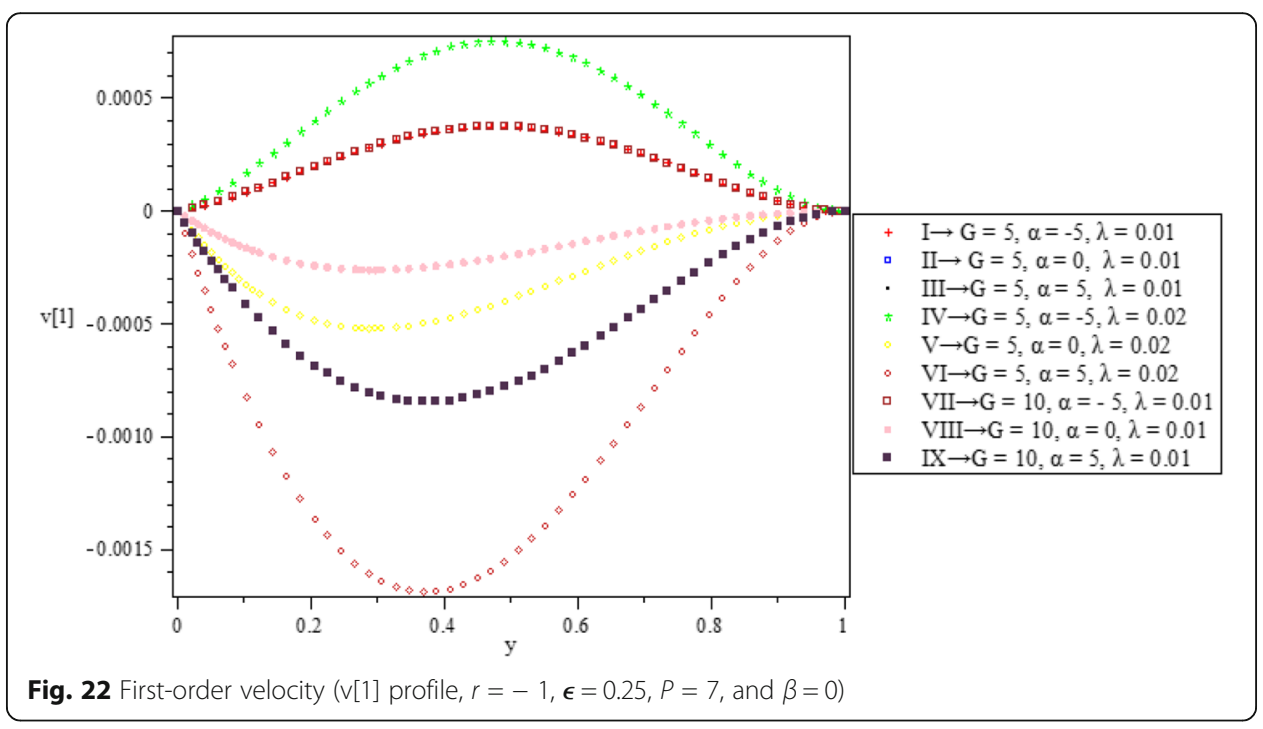




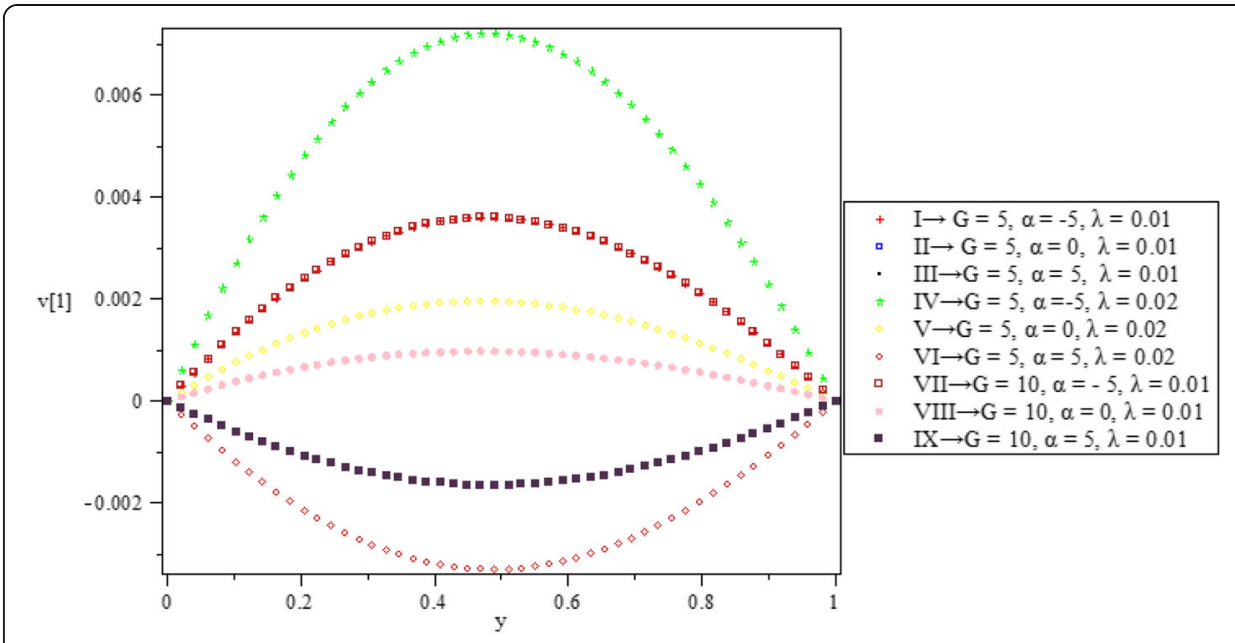

Fig. 23 First-order velocity (v[1] profile, $r=-1, \boldsymbol{\epsilon}=0.25, P=0.71$, and $\beta=1.5$ )

$(\alpha)$ increases on both walls. When $r=-1$ and Grashof number $G$ are increased from 5 to 10 , the skin friction decreases linearly as $\alpha$ increases from -5 to the value $\alpha=3$, then it increases again. While at $r=1$, the skin friction increases linearly and significantly as Grashof number $G$ increases on both walls.

\section{Temperature profile}

The behavior of the fluid temperature with changes in $\alpha$ is presented for $r=-1$ and $r=2$ respectively in Figs. 8 and 9. It is observed that the slip coefficient $\beta$ does not affect the zeroth-order temperature profile which gives results similar to that earlier obtained in Vajravelu and Sastri [10].

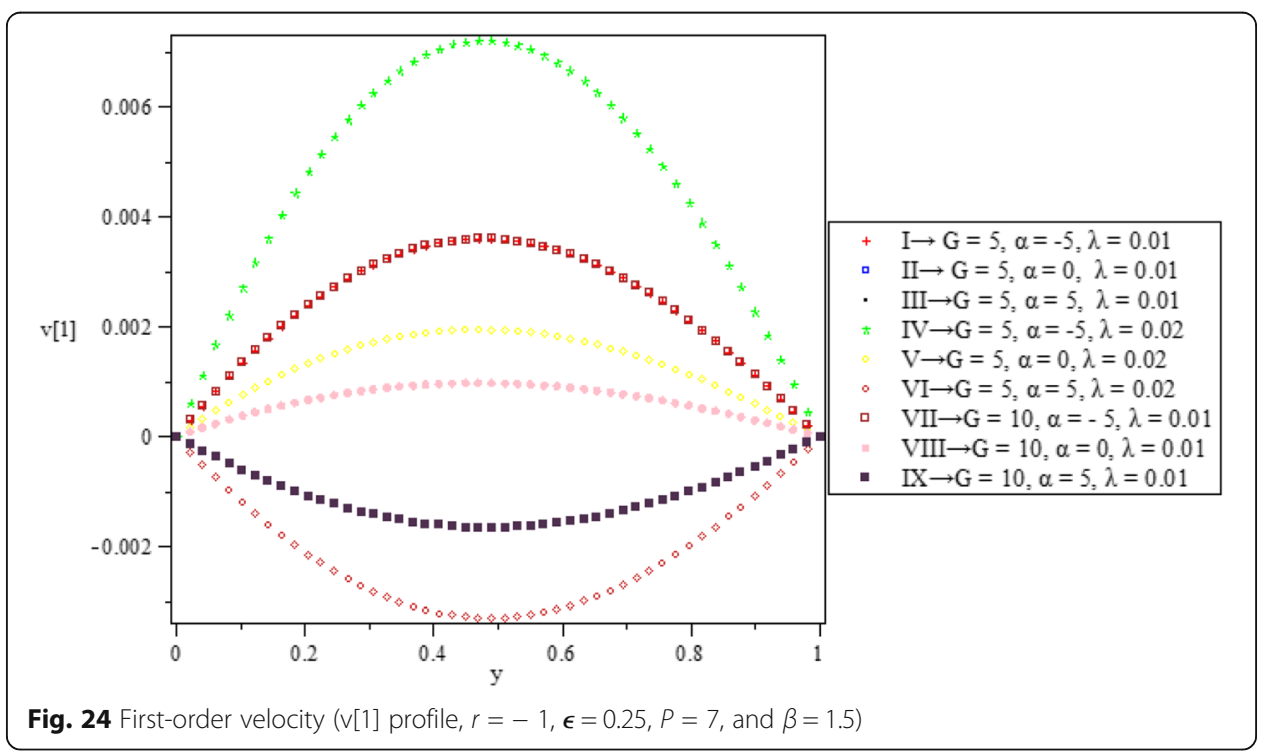




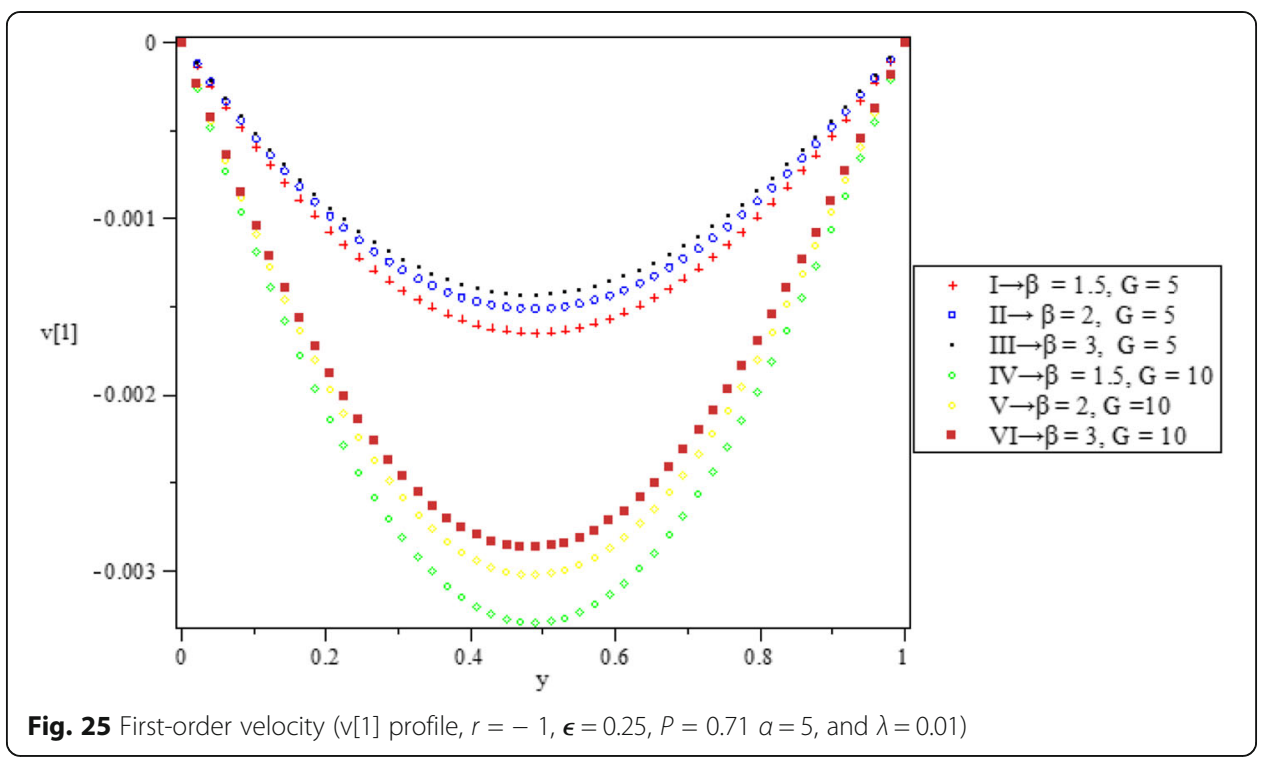

Also, the heat-transfer coefficient $N u_{0}$ is presented in Fig. 10. It can be seen that as $\alpha$ increases the heat transfer decreases on the wavy wall $y=0$ and the reversed holds on the flat wall $(y=1)$.

\section{Discussion of the first-order results}

Figures 11, 12, 13, 14, 15, 16, 17, 18, 19, 20, 21, 22, 23, 24, 25, 26, 27, 28, 29, 30, 31, 32, and 33 illustrate the behavior of the perturbed quantities (first-order solution) $u_{1}, v_{1}$, and $\theta_{1}$, when $m=-1$ and Prandtl number $(P)$ is 0.71 (air) and 7 (water).

\section{Velocity profile}

Figure 11 presents the behavior of perturbed velocity $u(1)$ when $r=1$ and the embedded slip parameter $\beta=0$. It is noticed that in the presence of heat sinks $\alpha<0$, the

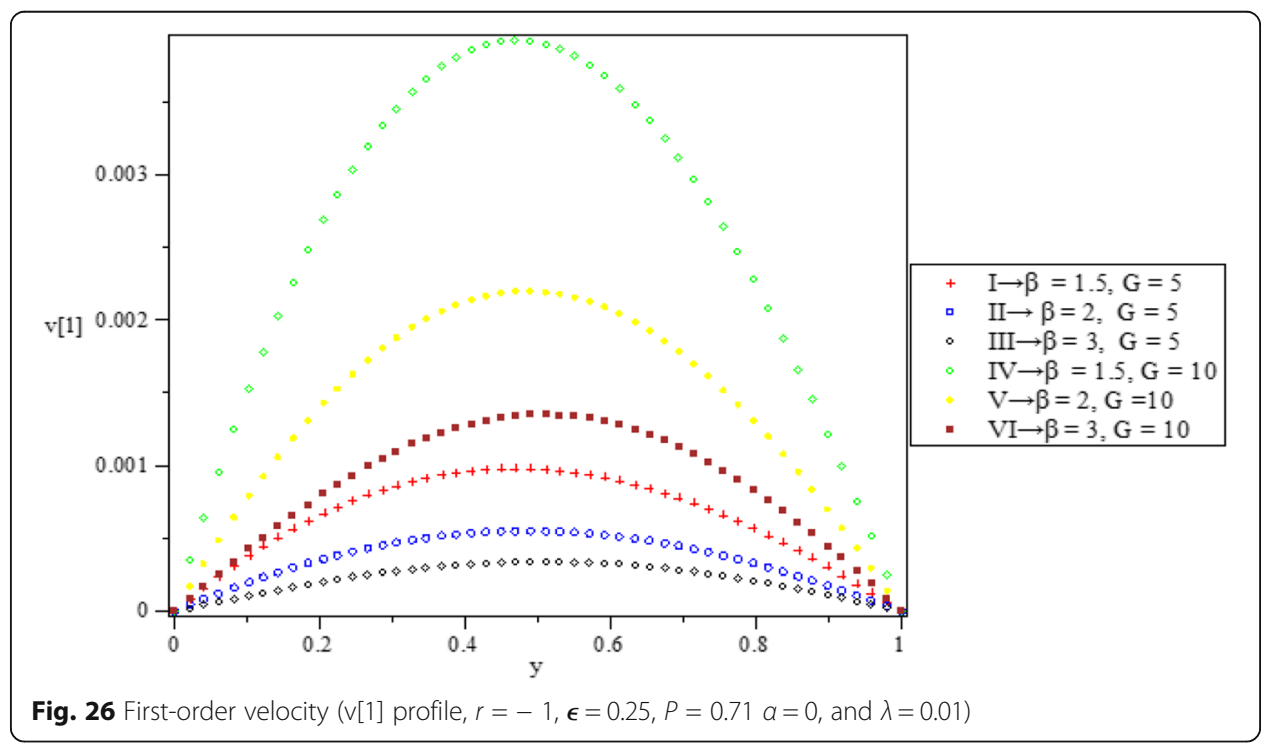




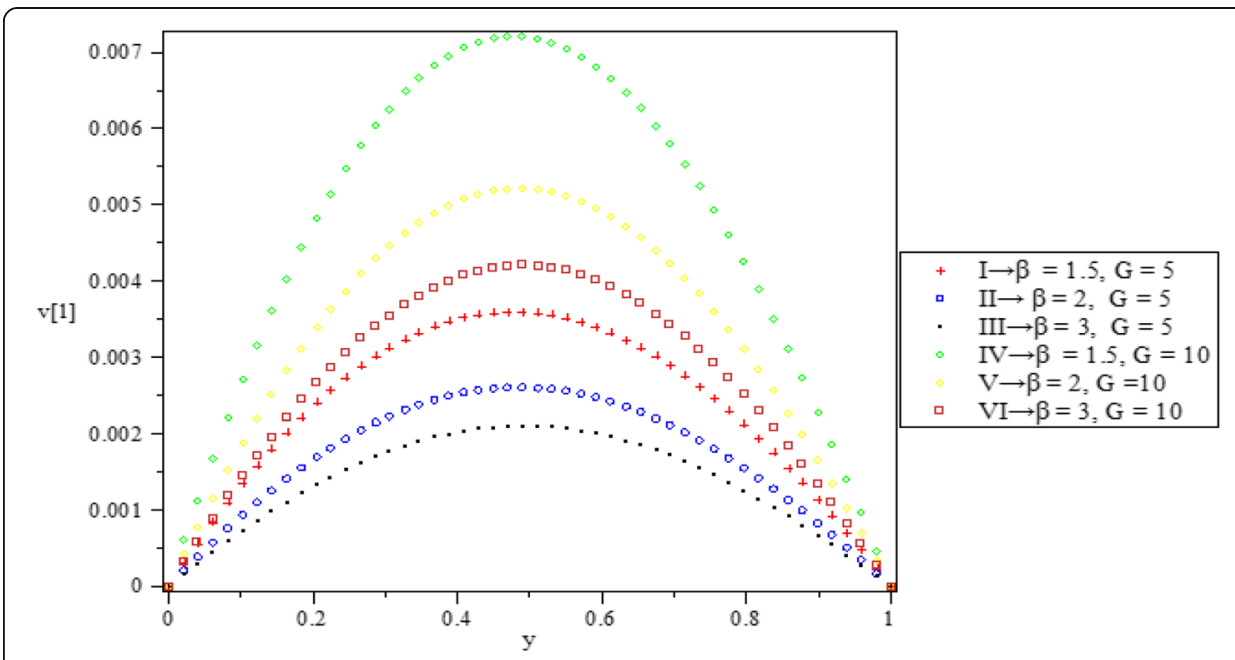

Fig. 27 First-order velocity (v[1] profile, $r=-1, \boldsymbol{\epsilon}=0.25, P=0.71 a=-5$, and $\lambda=0.01$ )

velocity $u(1)$ increases to a particular point of $y(y=0.55)$ and then decreases with constant $G$ and $\lambda$ (see curves I and II, VII and VIII). It is observed that there exists an increase in the velocity $u(1)$ up to $y=0.3$, followed by a decrease up to $y=0.7$ and finally increase for $y>0.7$ when $\alpha \geq 0$ (see curves II and III, XIII and IX). It is also realized that an increase in Grashof number $G$ or frequency parameter $\lambda$, the velocity increases close to the walls of the channel and decreases between the wall (see curves I and VII, II and XIII, III and IX) or (see curves I and IV, II and V, III and XI). However, it is observed that when Prandtl number $P=7$ (water), the behavior of the fluid velocity is the same for all cases of heat source $\alpha$ (see Fig. 12). Figures 13 and 14 show the behavior of the fluid velocity $u_{1}$ when there is a slip for both cases of $P=0.71$ (air) and $P=7$ (water). It is seen that the amplitude of the fluid velocity $u_{1}$ increases as the frequency parameter $\lambda$ or Grashof number $G$ increases. but the increase is more significant when $\alpha=-5$ than when $\alpha \geq 0$ (see curves I, IV, VII, and II, V, VIII, and III, VI, IX) on both figures. Physically, this indicates that higher values of $G$ enhance buoyancy force which

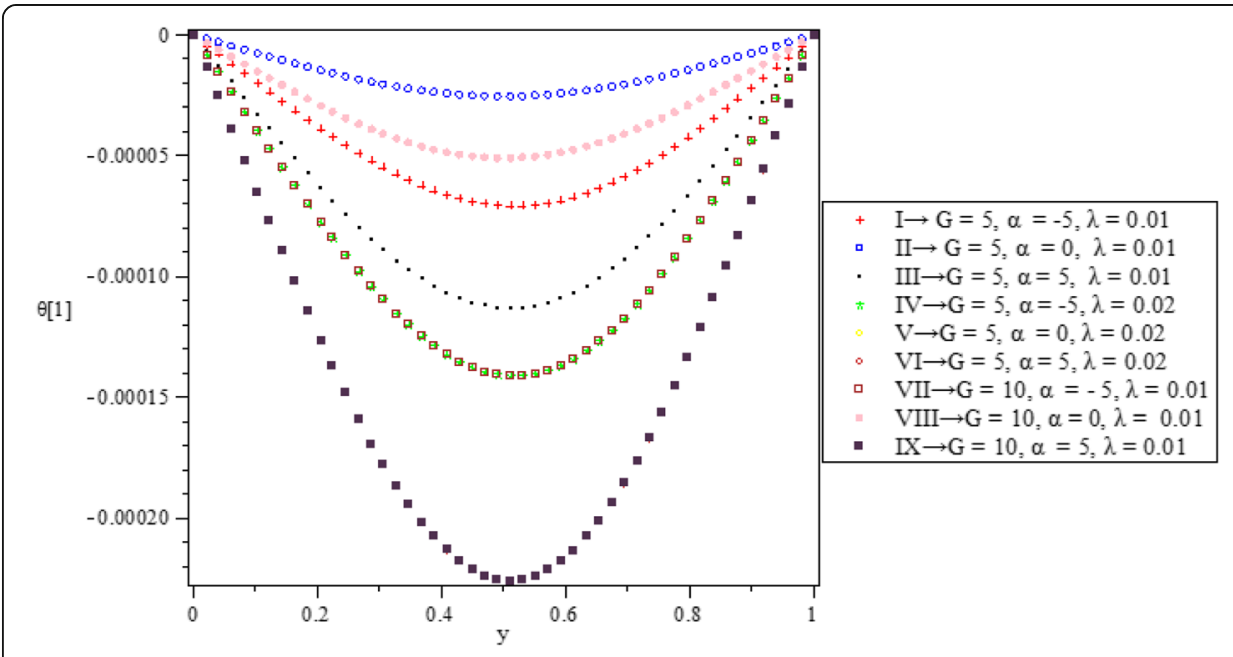

Fig. 28 First-order temperature ( $\theta[1]$ profile, $r=-1, \epsilon=0.25, P=0.71$, and $\beta=0$ ) 


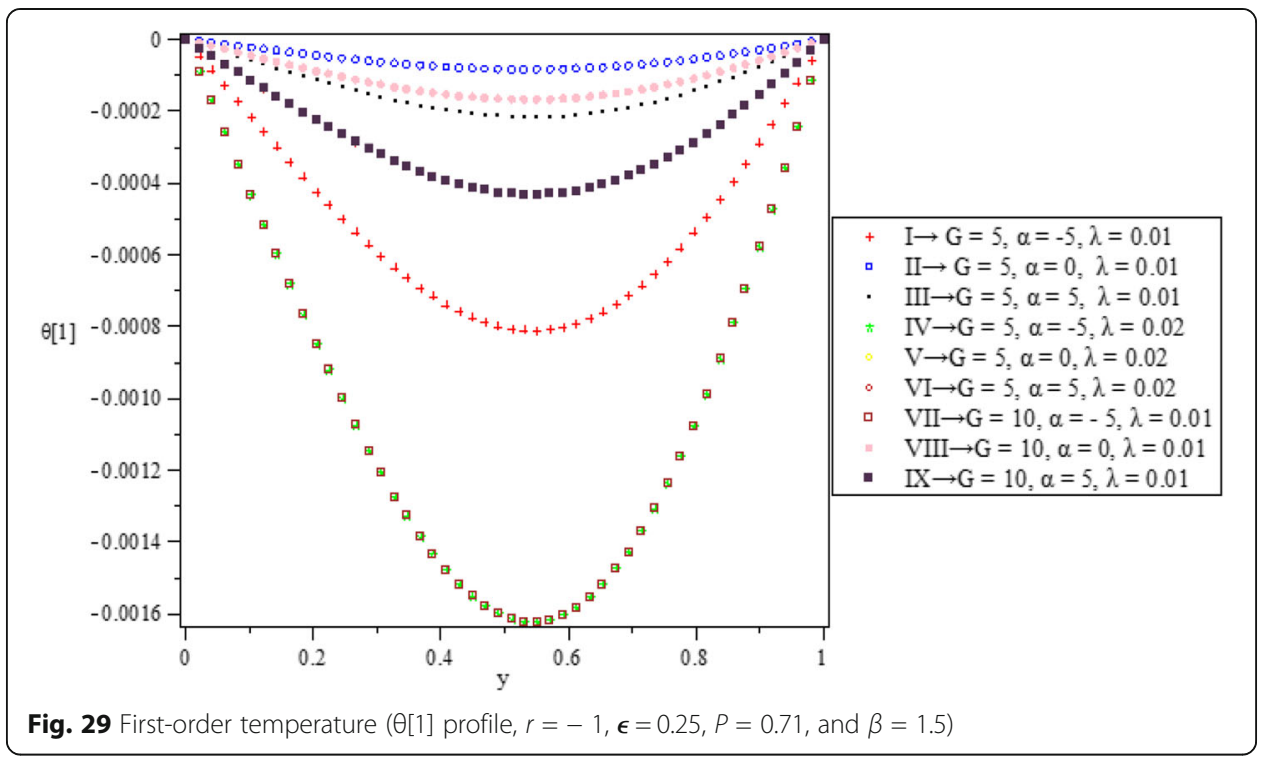

consequently within the channel, increases hydrodynamics. It is acknowledged that the positive value of $G$ corresponds to cooling the walls or heating the fluid by free convection current. Also, an increase in $P$ makes the thermal conductivity of the fluid to decrease. Thus at higher $P$, the rate at which the diffusion of heat takes place at the heated surface is more rapid.

Figures 15,16 , and 17 depict the effect of slip on the fluid velocity $u_{1}$ as the Grashof number $G$ varies between 5 and 10. It is observed that as the slip $(\beta)$ increases, the fluid velocity decreases and as the Grashof number $G$ increases, the amplitude of fluid $u_{1}$ increases. Physically, this means that when there is an increase in the slip condition $(\beta)$, the fluid becomes more viscous and resulted in a reduction in fluid velocity. However, the momentum boundary layer thickness decreases as $(\beta)$ increases.

Figures 18, 19, and 20 illustrate the effect of slip $\beta$ on the fluid velocity as the frequency $(\lambda)$ varies between 0.01 and 0.02 . It clearly shows that as $\beta$ increases, the velocity $u_{1}$ decreases at the flat wall. And as $\lambda$ increases, the velocity at the flat wall

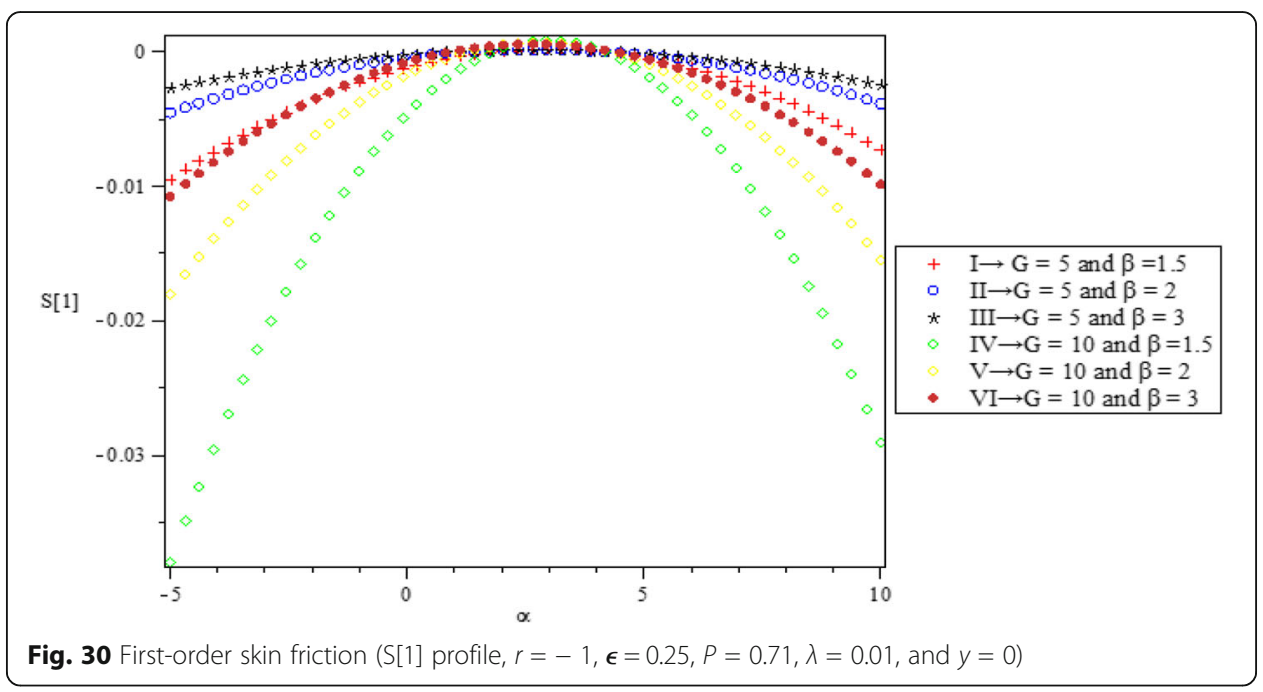




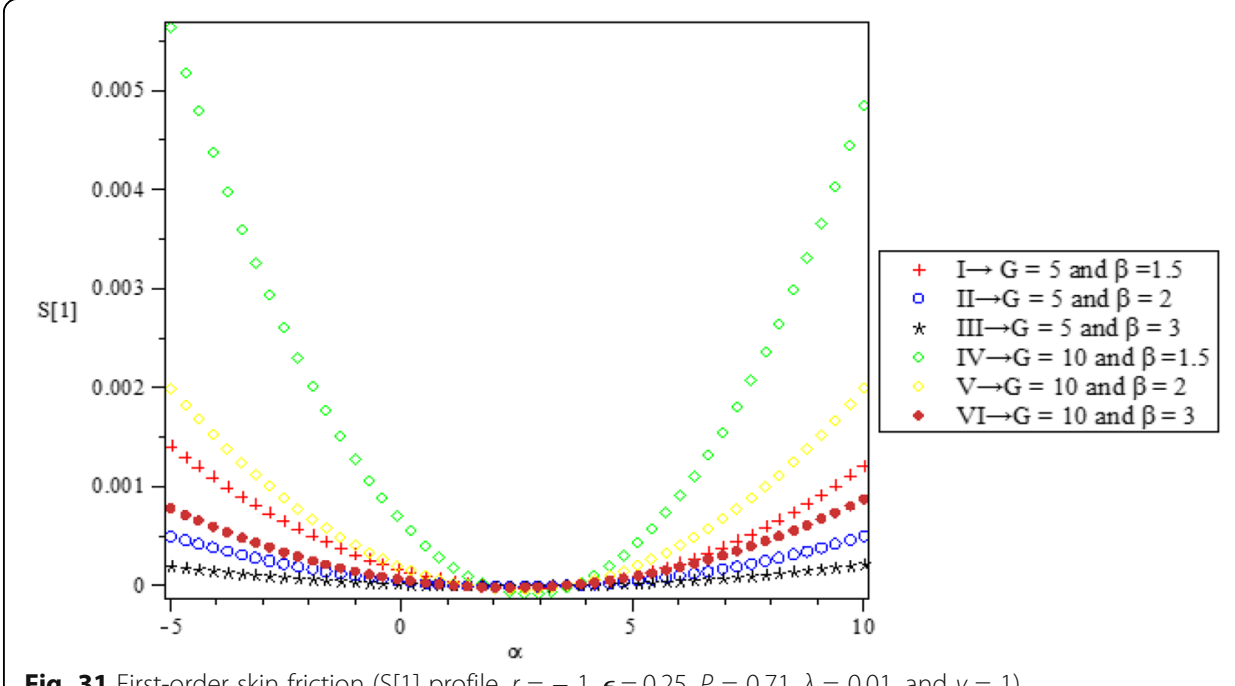

Fig. 31 First-order skin friction (S[1] profile, $r=-1, \boldsymbol{\epsilon}=0.25, P=0.71, \lambda=0.01$, and $y=1$ )

increases. Figures 21 and 22 show the behavior of the fluid velocity $v_{1}$ perpendicular to the channel length, when $\beta=0$ (i.e., no-slip condition). It is seen that as the heat source parameter $\alpha$ increases from -5 to 5 , the fluid velocity $v_{1}$ decreases significantly (see curves I, II, and III of Figs. 21 and 22). It is also noticed that with an increase of the Grashof number $G$ from 5 to 10 , the velocity is unaffected but with an increase in the frequency parameter $\lambda$ from 0.01 to 0.02 , there is an increase in the magnitude of the fluid velocity $v_{1}$. Figures 23 and 24 depict the effect of slip $\beta$ on the fluid velocity $v_{1}$ with the variation of the heat source $(\alpha)$, frequency $(\lambda)$, and Grashof number $G$ respectively. An increase in $\lambda$ gives a significant increase in the magnitude of the fluid velocity $\left(v_{1}\right)$, while an increase in the $\alpha$ reduces the fluid velocity $\left(v_{1}\right)$ profile. Physically, this implies that the presence of heat absorption coefficient $(\alpha)$ is capable of reducing the temperature, as a result, the thermal buoyancy effect decreases leading to a net reduction in fluid velocity.

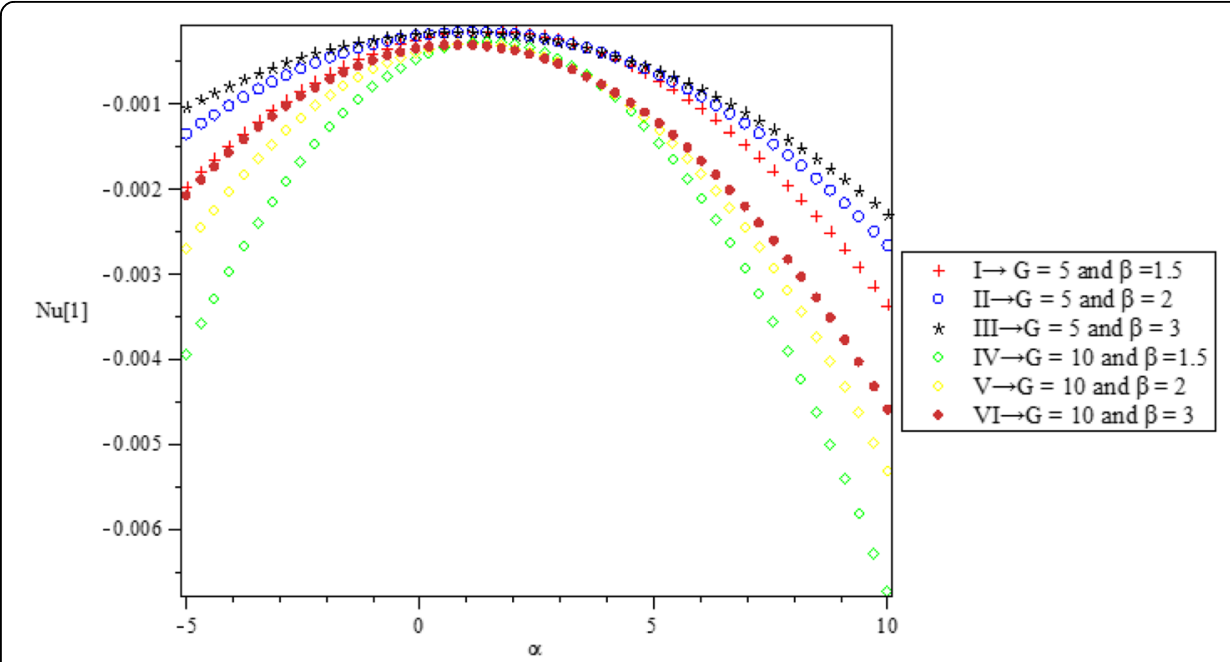

Fig. 32 First-order Nusselt number (Nu[1] profile, $r=-1, \boldsymbol{\epsilon}=0.25, P=0.71, \lambda=0.01$, and $y=0$ ) 


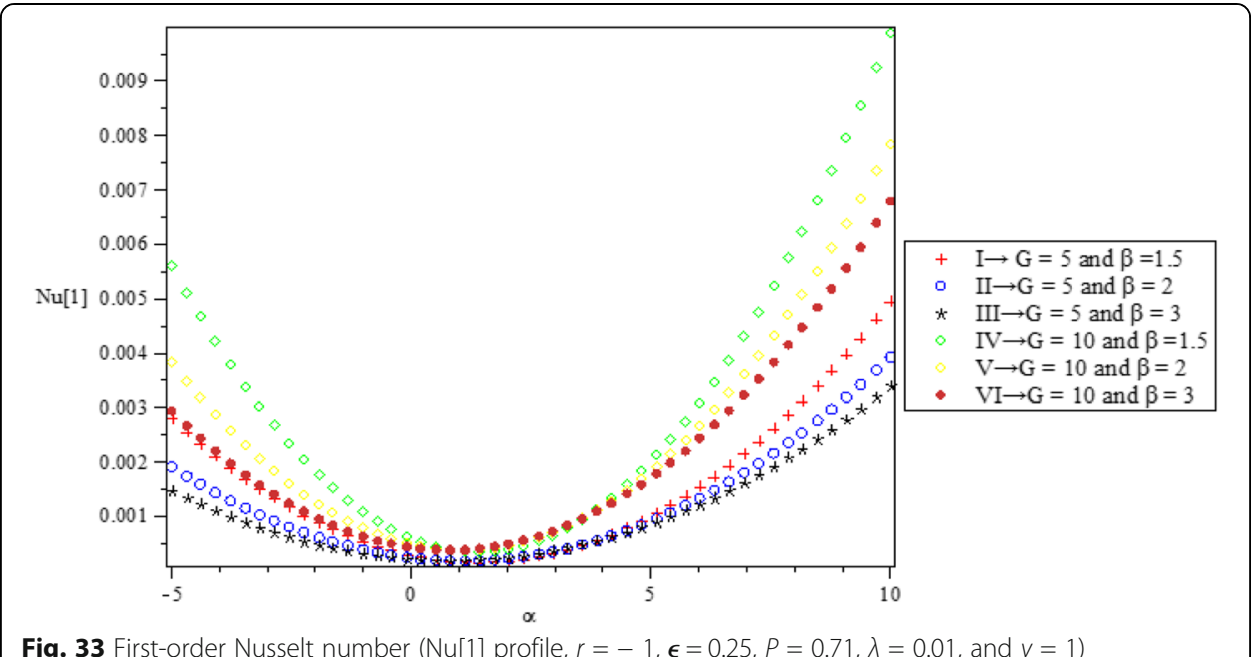

Figures 25,26 , and 27 illustrate the effect of slip $(\beta)$ with variation of heat source parameter $(\alpha)$ and Grashof number $G$. It is observed that increasing the slip $(\beta)$, the magnitude of the velocity of the fluid reduces in all the cases.

Figures 28 and 29 show the behavior of the fluid temperature $\theta_{1}$ when $\beta=0$ and $\beta=$ 1.5 respectively. It is observed that the $\operatorname{slip}(\beta)$ increases the magnitude of the temperature $\theta_{1}$ of the fluid comparing the two figures and by close observation it shows that the slip decreases the temperature of the fluid

Figures 30 and 31 show the effect of slip $(\beta)$ on skin friction at the walls of the channel. At the way wall $(y=0)$, the skin friction is an increasing function of $\alpha$, while at the flat wall $(y=1)$ is a decreasing function of $\alpha$. Also, at the wavy wall $(y=0)$, as the slip $(\beta)$ increases, the skin friction increases, while at the flat wall $(y=1)$, the reverse holds. Figures 32 and 33 illustrate the effect of slip $(\beta)$ on the Nusselt number $\left(N u_{1}\right)$, at the wavy wall (Fig. 32), the rate of heat transfer is parabolic and is an increasing function of $\alpha$. Also, when the slip $(\beta)$ increases, the rate of heat transfer at the wavy wall increases. From Fig. 33 which presents the heat transfer behavior at the flat wall. It is seen that the behavior is the reverse of the wavy wall.

\section{Conclusion}

An analysis is carried out on the effects of Navier slip on a steady flow of an incompressible viscous fluid confined within a spirally enhanced channel. The solution to the dimensionless governing equations was obtained using the Adomian decomposition method with the MAPLE 18 software. The effects of various parameters (such as G, $\beta$, $\lambda$ ) on fluid physical properties (i.e., velocity and temperature) were examined and illustrated graphically. The study brings out the following results of physical interest:

- The zeroth-order velocity $u_{0}$ increases linearly with an increase in Grashof number $G$ or heat source/sink parameter $\alpha$ (i.e., the velocity is directly proportional to $G$ or $\alpha$ ) when slip condition parameter $\beta=1.5$ for both cases of temperature ratio $r$.

- An increase in Grashof number $G$ or frequency parameter $\lambda$ leads to an increase in velocity $v_{1}$ profiles while the reverse is the case for an increase in slip parameter $\beta$. 
- The velocity $v_{1}$ profiles have an increasing influence on the frequency parameter $\lambda$ while an increase in heat source parameter $\alpha$ reduces the fluid velocity for $\beta=1.5$.

- The slip condition parameter $\beta$ does not have any effect on zeroth-order temperature $\theta_{0}$ while it increases the amplitude of the first-order temperature $\theta_{1}$ profiles.

- At the wavy wall $y=0$, the skin friction is an increasing function of $\alpha$ while a decrease is noticed at the flat wall $y=1$.

- The rate of heat transfer $N u_{1}$ at the wavy wall increases with an increase in the slip parameter $\beta$, but a decrease at the flat wall.

\section{Acknowledgements}

Not applicable.

\section{Availability of materials}

Data sharing is not available to this article as no data set were generated or analyzed during the current study.

\section{Authors' contributions}

J.A.G. and J.U.A. modelled the problem and drew the diagram of the flow channel. J.A.G., J.U.A., and T.L.O. introduced the dimensionless variables and transformed the modelled problem into dimensionless form. J.U.A. and T.L.O. solved the problem numerically and computed the results. J.A.G., J.U.A., and T.L.O. discussed the results with conclusions. All authors read and approved the final manuscript.

\section{Funding}

No specific funding received for this work.

\section{Competing interests}

The authors declare that they have no conflict of interest.

\section{Author details}

${ }^{1}$ University of Ilorin, Ilorin, Kwara State, Nigeria. ${ }^{2}$ College of Education, Oro, Kwara State, Nigeria.

Received: 2 December 2019 Accepted: 13 April 2020

Published online: 15 June 2020

\section{References}

1. Chaudhary, D., Singh, H., Jain, N.C.: Effects of Hall current and thermal radiation on an unsteady free convection slip flow along a vertical plate embedded in a porous medium with constant heat and mass flux. AMP. 1(2), 11-26 (2013)

2. Derek, C., Tretheway, D.C., Meinhart, C.D.: Apparent fluid slip at hydrophobic microchannel wall. APF. 14, L9-L12 (2002)

3. Mehmood, A., Ali, A.: The effect of slip condition on unsteady MHD oscillatory flow of a viscous fluid in a planar channel. Rom.Journal. Phys. 52(1-2), 85-91 (2007)

4. Adesanya, O., Gbadeyan, J.A.: Adomian decomposition approach to steady visco-elastic fluid flow with slip through a planar channel. Int J. of non-linear sci. 9(2), 86-94 (2010)

5. Halima, U., Fazle, M., Giulio, L.: Heat and mass transfer along vertical channel in porous medium with radiation effect and slip condition. International Journal of Heat and Technology (IJHT). 34(1), 129-136 (2016)

6. Gbadeyan, J.A., Dada, M.S.: On the influence of radiation and heat transfer on an unsteady MHD non-Newtonian fluid flow in a porous medium. JMR. 5(3), 40-50 (2013)

7. Makinde, O.D.: Computational modelling of MHD unsteady flow and heat transfer toward a flat plate with Navier slip and Newtonian heating. BJCE. 29(1), 159-166 (2012)

8. M. Mahmoud, S. Waheed, Effect of slip and heat generation absorption on MHD mixed convection flow of a micro polar fluid over a heated stretching surface, MPE, volume 2010, Article ID 579162, 20 pages doi:https://doi.org/10.1155/ 2010/579162

9. Martin, M.J., Boyd, I.D.: Momentum and heat transfer in a laminar boundary layer with slip flow. JTHT. 20(4), 710-719 (2016)

10. Varjravelu, K., Sastri, K.S.: Free convection heat transfer in a viscous incomoressible fluid confined between a long vertical wavy wall and a paralle flat wall. J. F M. 86, 365-383 (1978)

11. Fasogbon, P.F.: Bouyancy driven flow in a rough channel. J. Application Functional Differential Equation (JAFDE). 1(1), 45-58 (2006)

12. Fasogbon, P.F., Omolehin, J.O.: Radiation effect on natural convection in spirally enhanced channel. JEMTA. 3(1), 1-28 (2008)

13. Fasogbon, P.F.: Analytical studies of heat and mass transfer by convection in a two dimensional irregular channel. Int.J.of Appl.Math. and Mech. 6(4), 17-37 (2010)

14. Dada, M.S., Disu, A.B.: Heat transfer with radiation and temperature dependent heat source in MHD free convection flow in a porous medium between two vertical wavy walls. J. of Nig. Math. Soc. 34(2), 200-215 (2015)

15. Gbadeyan, J.A., Oyekunle, T.L., Abubakar, J.U.: Thermal radiation and chemical reaction effects on free convective heat and mass transfer flow through an irregular channel. FUW Trends in Science and Technology Journal. 2(1B), 462-477 (2017) 
16. Gbadeyan, J.A., Oyekunle, T.L., Fasogbon, P.F., Abubakar, J.U.: Soret and Dufour effects on heat and mass transfer in chemically reacting MHD flow through a wavy channel. JTUS. 12(5), 631-651 (2018). https://doi.org/10.1080/16583655. 2018.1492221

17. Teneja, R., Jain, N.C.: MHD flow with slip effect and temperature dependent heat source in a viscous incompressible fluid confined between a long vertical wavy wall and a parallel flat wall. DSJ. 54(1), 21-29 (2014)

18. Kumar, R., Chand, K.: Effect of slip condition and wall hall current on a steady MHD flow visco-elastic fluid past an infinite vertical porous plate through porous medium. IJST. 3(4), 3124-3134 (2011)

19. Younghae, D.O., RAMESH, G.K., ROOPA, G.S., SANKAR, M.: Navier's slip condition on time dependent Darcy-Forchheimer nanofluid using spectral relaxation method. JCSU. 26(7), 2000-2010 (2019)

20. Gbadeyan, J.A., Titiloye, E.O., Adeosun, A.T.: Effect of variable thermal conductivity and viscosity on casson nanofluid flow with convective heating and velocity slip. Heliyon. 6, 1-10 (2020)

21. Chen, W., Lu, Z:: An algorithm for Adomian decomposition method. AMC. 159, 221-235 (2004)

\section{Publisher's Note}

Springer Nature remains neutral with regard to jurisdictional claims in published maps and institutional affiliations.

Submit your manuscript to a SpringerOpen ${ }^{\odot}$ journal and benefit from:

- Convenient online submission

- Rigorous peer review

- Open access: articles freely available online

- High visibility within the field

- Retaining the copyright to your article 